\title{
Steady state harmonic response of nonlinear soil-structure interaction problems through the Preisach formalism
}

\author{
by
}

\author{
Pierfrancesco Cacciola $^{1}$ and Alessandro Tombari ${ }^{1}$ \\ ${ }^{1}$ School of Environment and Technology, University of Brighton, Cockcroft Building, Brighton
}

\begin{abstract}
Steady state harmonic response of nonlinear soil-structure interaction problems is addressed in this paper. Due to well-known stiffness degradation phenomenon, the steady-state study is referred to small-medium strain condition whereas no significant pore water pressure change is observed (e.g. clayey soils and sands in drained conditions). A novel cyclic hysteretic model based on the Preisach formalism is proposed to describe the cyclic behavior of soil-foundation interaction. Through a harmonic balance procedure, furthermore, the steady state response of nonlinear soil-structure interaction problems is determined. Equivalent amplitude-dependent stiffness and damping have been derived in closed form to reliably describe the nonlinear soil-structure interaction phenomenon. Those expressions are as a function of the elastic foundation stiffness and horizontal force and moment capacity of the foundation. Furthermore, handy expressions of equivalent fundamental period and damping also dependent from the level of the excitation are also derived. The proposed hysteretic model, moreover, has the advantage to be easily calibrated to match modulus reduction and damping curves determined from numerical or experimental tests. Numerical applications and comparisons with more advanced models and experimental data available in literature are also presented.
\end{abstract}

Keywords: Nonlinear soil-structure interaction, Preisach model, soil hysteresis, harmonic balance. 


\section{$1 \quad$ Introduction}

Soil-structure interaction (SSI) studies can be tracked back to the late 19th century and a recent review of the main achievements can be found in the paper by Kausel [1]. Earlier contributions, still used nowadays by researchers and practitioners, are based on the study of the response of an infinite (or semi-infinite) elastic medium under either imposed loads or imposed displacements. A collection of those solutions is presented in the book from Poulos and Davis [2] and in the Dobry and Gazetas [3] paper. Due to the nonlinear behaviour of the soil medium the nonlinear soil-structure interaction problem has been investigated in the last five decades. It has to be pointed out that the assumption of linear soil-structure interaction may not be always beneficial and might differ significantly from the nonlinear case $[4,5]$. To date three main approaches are used for the analyses of nonlinear soil structure interactions problems (see e.g. Jakub and Roesset, [6]): i) linear analysis using soil properties consistent with the expected level of strains in the soil with the consequent use of an estimated reduced shear modulus and increased damping; ii) iterative linear analyses using soil properties (modulus and damping) adjusted to match assigned shear modulus reduction and damping curves; iii) nonlinear analysis in the time domain using a discrete model (e.g. finite elements or finite differences) with appropriate nonlinear constitutive equations for the soil. In this regard, the sustained development of computational tools allows researchers and practitioners to consider various complicated phenomena in their analyses. Major drawback of this approach is the high computational cost and the difficulty to calibrate accurately advanced FE models with experimental data. A comparison between equivalent linear and fully nonlinear SSI analysis has been recently carried on by Bolisetti et al. [4]. In the framework of Finite Element approach for nonlinear SSI, computational aspects are generally addressed through reduced domain models see e.g Santisi D'Avila and LopezCaballero [7] or through macro elements (see e.g. Pecker and Chatzigogos [8], Chatzigogos et al [9], Figini et al [10], Cavalieri et al [11]). Despite the high level of accuracy of FE models, simplified 
models in SSI study still remain attractive, as allow practitioners and researches, with a minimum computational effort, to perform parametric analyses and to develop a feel for the physical meaning and relative importance of the various factors, with more personal control of calculations (Dobry [12]). In this regard, non-linear Winkler models representing the cyclic soil behavior have been widely used for different applications. Specifically, Boulanger et al. [13] studied soil-pile foundation interaction through the dynamic Beam-on-Nonlinear-Winkler-Foundation model. Subsequently, this approach has been extended by Raychowdhury and Hutchinson [14] to shallow foundations and applied to nuclear reactors in [15]. An analytical approach has been proposed in [16] to evaluate the moment-rotation response for both uplift- and yield-only conditions of a rigid foundation on a Winkler soil model. This approach has been extended by Allotey and El Naggar [17] to provide a complete analytical solution for the static moment-rotation response of a rigid foundation resting on a Winkler soil model. A generalized Winkler model for the behavior of shallow foundations has been proposed by Houlsby et al. [18]. In the last decade a growing body of literature has been devoted to simplified models and the calibration of nonlinear springs able to capture the main features of nonlinear SSI such as the equivalent period and foundation damping. In this regard, nonlinear rocking stiffness have been determined by Gazetas et al. [19] through an empirical approach based on FE analyses conducted on rocking foundations for various foundation shapes considering homogeneous clay under undrained conditions. This approach has been extended by Anastasopoulos and Kontoroupi [20] and applied to motorway bridges in [21]. A nonlinear sway-rocking model has been developed in [22]. Recently, a study on the stiffness degradation and damping of pile foundations under dynamic loadings has been performed in [23] by calibrating nonlinear translational and rotational springs through experimental data from centrifuge tests. The above simplified models have been used either to determine equivalent fundamental periods for different level of excitations of the nonlinear SSI systems [19] or to determine the whole-time domain response [20-22]. Moved by the 
necessity to gather relevant dynamic information for design purposed Ahmadi et al [24] used an equivalent linearization approach to MDOF system with nonlinear Coulomb-type SSI springs and also highlighted the lack of simplified studies in this regard for nonlinear soil-structure interaction problems.

In this paper, the steady state response for nonlinear SSI problems is investigated with the aim to provide simple basic information useful for design purpose. Steady state dynamic response is manifested in soil-structure interaction problems whereas no significant pore water pressure change is observed, such as for small-medium strain in clayey soils and sands in drained conditions (see e.g. Li et al. [23] and Vucetic and Mortezaie [25]) and therefore the dependency of stiffness and damping from the number of cycles can be neglected.

In this regard, the harmonic balance approach originally proposed by Iwan [26] and Spanos [27] is herein extended to nonlinear SSI problems to determine equivalent stiffness and damping coefficients both dependent from the response amplitude. The steady state response is readily determined by solving a system of nonlinear algebraic equations. Equivalent fundamental periods are also determined. Moreover, the Preisach model of hysteresis [28] widely adopted up until now in the framework of ferromagnetism, and more recently adopted to the study of hysteretic behaviour of structural and mechanical systems is adopted for the first time in this paper to model nonlinear soilstructure interaction problems. Preisach representation of hysteresis belongs to the class of model based on the distributed elements, which assume that hysteresis can be considered such as a superposition of simple hysteretic operators. A detailed description of the Preisach hysteresis representation can be found in $[29,30]$. The main feature of the Preisach model is the capability to adjust itself to various hysteretic phenomena and even capture nonsymmetric minor loops present in many physical systems. Various classical hysteretic model can be represented via the Preisach formalism. For instance, the Jenkins and the distributed Jenkins-Iwan models can be seen as particular 
case of the Preisach model of hysteresis. It is worth nothing, the Jenkins-Iwan model (known also as Iwan model) has been used for nonlinear soil-structure interaction in earlier contributions (see e.g. [4], [7], [8], and [31]), therefore suggesting the more general Preisach model can be also tailored for SSI studies. It has to be emphasized, due its versatility, the Preisach model of hysteresis is calibrated to match perfectly both stiffness and damping curves determined by experimental tests and/or available in literature, such those proposed in [19] and in [23]. Moreover, closed-form expressions of model parameters are suitably determined for Iwan-type hysteresis. Numerical results show the accuracy and the efficiency of the proposed approach.

\section{Problem formulation}

Consider the MDoF system depicted in Figure 1a. The superstructure is a linear behaving structure. The foundation is considered rigid and resting on a nonlinear compliant soil. The nonlinear soilstructure interaction mechanism is modelled trough two uncoupled nonlinear hysteretic springs as depicted in Figure 1b. The equations governing the motion of the structure undergoing forced vibrations can be cast in the following form:

$$
\boldsymbol{M} \ddot{\boldsymbol{u}}(t)+\boldsymbol{C} \dot{\boldsymbol{u}}(t)+\boldsymbol{K} \boldsymbol{u}(t)+\boldsymbol{h}(t)=\boldsymbol{p}(t)
$$

where $\boldsymbol{u}(t)$ is the vector collecting the $\mathrm{i}=1, \ldots, \mathrm{n}$ degrees of freedom of the system, $u_{i}, \boldsymbol{M}$ is the mass matrix listing the floor masses $m_{i}, \mathbf{C}$ is the damping matrix, $\boldsymbol{K}$ is the stiffness matrix collecting the floor stiffnesses $k_{i}$, and $\boldsymbol{h}(t)$ is the nonlinear hysteretic vector encompassing the nonlinear forces generated by the soil springs, i.e. the horizontal force, $f_{h}$ and the moment $f_{\theta}$, and $\boldsymbol{p}(t)$ is the loading vector. 


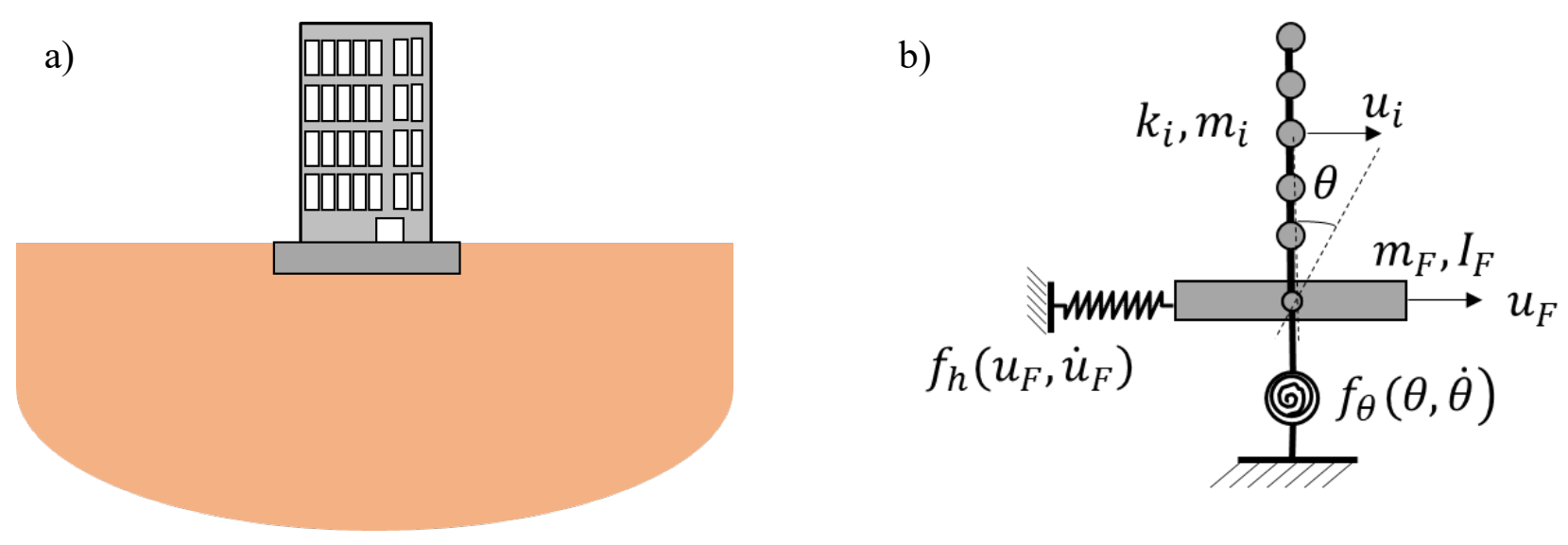

Figure 1: Structure resting over a non-linear compliant soil a) and its discretization b)

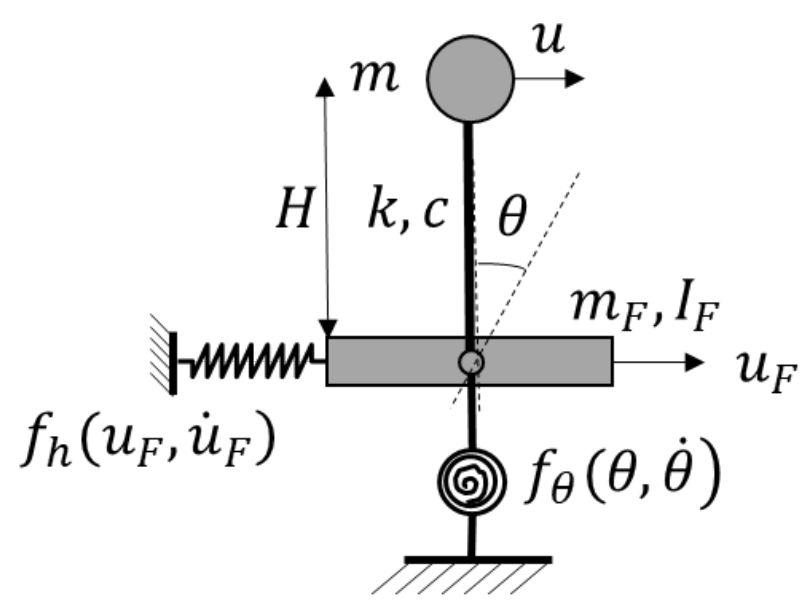

Figure 2: 3-DoF nonlinear soil-structure system

For the simplest case (Figure 2) of 3-DoF nonlinear soil-structure coupled system $($ i.e. $\boldsymbol{u}(t)=$ $\left[u u_{F} \theta\right]^{T}$ ) those matrixes reduces to

$$
\boldsymbol{M}=\left[\begin{array}{ccc}
m & 0 & 0 \\
0 & m_{F} & 0 \\
0 & 0 & I_{F}
\end{array}\right]
$$

where $m$ is the superstructure mass, $m_{F}$ is the foundation mass, $I_{F}$ is the foundation moment of inertia and 


$$
\boldsymbol{K}=\left[\begin{array}{ccc}
k & -k & -k H \\
-k & k & k H \\
-k H & k H & k H^{2}
\end{array}\right]
$$

in which $k$ is the superstructure lateral stiffness and $H$ the height of the superstructure. Also,

$$
\boldsymbol{C}=\left[\begin{array}{ccc}
c & -c & 0 \\
-c & c & 0 \\
0 & 0 & 0
\end{array}\right]
$$

with $c$ the viscous damping of the superstructure. It is noted the damping matrix (4) can be tailored to consider additional mechanism of dissipation of energy such as the radiation damping, herein omitted for simplicity's sake. The hysteretic term can be written as

$$
\boldsymbol{h}(t)=\left[\begin{array}{lll}
0 & f_{h}\left(u_{F}, \dot{u}_{F}\right) & f_{\theta}(\theta, \dot{\theta})
\end{array}\right]^{T}
$$

where $f_{h}\left(u_{F}, \dot{u}_{F}\right)$ and $f_{\theta}(\theta, \dot{\theta})$ are the nonlinear hysteretic elements pertinent to the translational and rotational foundation degrees of freedom $u_{F}$ and $\theta$, respectively.

Once defined a pertinent model of the hysteretic elements (see e.g. reference [19-23]) the time domain solution can be readily performed through various numerical algorithms such as the Runge-Kutta, Hilber-Hughes-Taylor or Newmark methods. For design purposes, however, it might be useful to determine the steady-state response, the equivalent period and the damping useful for parametric studies that do not require the full step-by-step time history response.

\section{Steady-state harmonic response}

In this section, the steady-state harmonic response of the soil-structure systems governed by Eq. (1) is addressed. Despite this approach has been successfully applied in the last 5 decades to mechanical and structural systems manifesting hysteresis ([26], [27]), to the authors' best knowledge it has not been applied yet to nonlinear SSI problems. Due to the mathematical structure of Eq. (1) the statestate harmonic response can be readily determined. To this aim, the loading term in Eq. (1) is assumed 
to be a harmonic excitation, i.e. $\mathbf{p}(t)=\boldsymbol{p}_{0} \cos (\omega t)$, with $\boldsymbol{p}_{0}$ the vector listing the input amplitudes, $\omega$ the circular frequency and $t$ is the time. The approach herein adopted assumes that the solution exhibits a pseudo-harmonic behavior, thus the response foundation degrees of freedom and their velocities can be written in the form

$$
u_{F}(t)=a_{h}(t) \cos (\omega t+\phi(t)), \quad \dot{u}_{F}(t)=-a_{h}(t) \omega \sin (\omega t+\phi(t)),
$$

and

$$
\theta(t)=a_{\theta}(t) \cos (\omega t+\phi(t)), \quad \dot{\theta}(t)=-a_{\theta}(t) \omega \sin (\omega t+\phi(t))
$$

in which the amplitudes $a_{h}(t)$ and $a_{\theta}(t)$ and the phase $\phi(t)$ are assumed slowly varying with respect to time. According to this assumption, the nonlinear terms $f_{h}\left(u_{F}, \dot{u}_{F}\right)$ and $f_{\theta}(\theta, \dot{\theta})$ are appropriately replaced, in a harmonic balance sense, by the expressions (Spanos [27], Spanos et al. [33-34])

$$
f_{h}\left(u_{F}, \dot{u}_{F}\right)=c_{e, h}\left(a_{h}\right) \dot{u}_{F}+k_{e, h}\left(a_{h}\right) u_{F},
$$

where

$$
c_{e, h}\left(a_{h}\right)=-\frac{1}{\pi \omega a_{h}} \int_{0}^{2 \pi} f_{h}\left(a_{h} \cos \vartheta,-a_{h} \omega \sin \vartheta\right) \sin \vartheta d \vartheta
$$

and

$$
k_{e, h}\left(a_{h}\right)=\frac{1}{\pi a_{h}} \int_{0}^{2 \pi} f_{h}\left(a_{h} \cos \vartheta,-a_{h} \omega \sin \vartheta\right) \cos \vartheta d \vartheta
$$

are the equivalent horizontal damping and stiffness, respectively. Similar expressions are determined for the rotational hysteretic element, that is:

$$
f_{\theta}(\theta, \dot{\theta})=c_{e, \theta}(\theta) \dot{\theta}+k_{e, \theta}(\theta) \theta
$$

where

$$
c_{e, \theta}\left(a_{\theta}\right)=-\frac{1}{\pi \omega a_{\theta}} \int_{0}^{2 \pi} f_{\theta}\left(a_{\theta} \cos \vartheta,-a_{\theta} \omega \sin \vartheta\right) \sin \vartheta d \vartheta
$$


and

$$
k_{e, \theta}\left(a_{\theta}\right)=\frac{1}{\pi a_{\theta}} \int_{0}^{2 \pi} f_{\theta}\left(a_{\theta} \cos \vartheta,-a_{\theta} \omega \sin \vartheta\right) \cos \vartheta d \vartheta
$$

Therefore, the equivalent linear system of Eq. (1) is

$$
\boldsymbol{M} \ddot{\boldsymbol{u}}(t)+\left(\boldsymbol{C}+\boldsymbol{C}_{e}\left(a_{h}, a_{\theta}\right)\right) \dot{\boldsymbol{u}}(t)+\left(\boldsymbol{K}+\boldsymbol{K}_{e}\left(a_{h}, a_{\theta}\right)\right) \boldsymbol{u}(t)=\boldsymbol{p}_{0} \cos (\omega t)
$$

where $\boldsymbol{K}_{e}\left(a_{h}, a_{\theta}\right)$ and $\boldsymbol{C}_{e}\left(a_{h}, a_{\theta}\right)$ are the equivalent stiffness and equivalent damping matrixes, respectively. For the 3DoF system given in Figure $2, \boldsymbol{K}_{e}\left(a_{h}, a_{\theta}\right)$ and $\boldsymbol{C}_{e}\left(a_{h}, a_{\theta}\right)$ assume the following form:

$$
\boldsymbol{K}_{e}\left(a_{h}, a_{\theta}\right)=\left[\begin{array}{ccc}
0 & 0 & 0 \\
0 & k_{e, h}\left(a_{h}\right) & 0 \\
0 & 0 & k_{e, \theta}\left(a_{\theta}\right)
\end{array}\right]
$$

and

$$
\boldsymbol{C}_{e}\left(a_{h}, a_{\theta}\right)=\left[\begin{array}{ccc}
0 & 0 & 0 \\
0 & c_{e, h}\left(a_{h}\right) & 0 \\
0 & 0 & c_{e, \theta}\left(a_{\theta}\right)
\end{array}\right]
$$

Roto-translational coupling can be also included through pertinent off-diagonal terms whereas necessary. The steady-state response can be now readily determined by the meaning of the frequencyresponse function given by the equation

$$
\mathbf{U}(\omega)=\left[\left(\boldsymbol{K}+\boldsymbol{K}_{e}\left(a_{h}, a_{\theta}\right)\right)+\left(\boldsymbol{C}+\boldsymbol{C}_{e}\left(a_{h}, a_{\theta}\right)\right)-\omega^{2} \boldsymbol{M}\right]^{-1} \boldsymbol{p}_{0}
$$

which represents a nonlinear algebraic equation, as the vector $\mathbf{U}(\omega)$ encompasses also the amplitudes $a_{h}$ and $a_{\theta}$, whose solution can be pursued by traditional numerical techniques such us the Newton's method. It is noted, by the meaning of Eq. (14) for the 3DoF system the equivalent fundamental period can be determined in closed form by solving the eigenvalue problem

$$
\left(\boldsymbol{K}+\boldsymbol{K}_{e}\left(a_{h}, a_{\theta}\right)\right) \boldsymbol{\Phi}\left(a_{h}, a_{\theta}\right)=\boldsymbol{M} \boldsymbol{\Phi}\left(a_{h}, a_{\theta}\right) \boldsymbol{\Omega}^{2}\left(a_{h}, a_{\theta}\right)
$$


where $\boldsymbol{\Phi}\left(a_{h}, a_{\theta}\right)$ and $\mathbf{\Omega}^{2}\left(a_{h}, a_{\theta}\right)$ are the modal and spectral matrices both functions of the amplitudes $a_{h}$ and $a_{\theta}$, respectively. For the particular case of massless rigid foundation (i.e. $m_{F}=I_{F}=0$ ) the solution of the eigenproblem (18) leads to

$$
\frac{1}{\omega_{e}^{2}\left(a_{h}, a_{\theta}\right)}=m\left(\frac{1}{k}+\frac{1}{k_{e, h}\left(a_{h}\right)}+\frac{H^{2}}{k_{e, \theta}\left(a_{\theta}\right)}\right)
$$

where $\omega_{e}\left(a_{h}, a_{\theta}\right)$ is the equivalent fundamental frequency. After simple algebra Eq. (19) can be written as

$$
\frac{T_{e}\left(a_{h}, a_{\theta}\right)}{T}=\sqrt{1+\frac{k}{k_{e, h}\left(a_{h}\right)}+\frac{k H^{2}}{k_{e, \theta}\left(a_{\theta}\right)}}
$$

in which $T$ is the fixed base period and $T_{e}\left(a_{h}, a_{\theta}\right)$ is the equivalent period of the nonlinear SSI system. Eq. (20) can be seen as the extension of the equivalent period determined by Veletsos and Meek [34] to the case of nonlinear SSI systems. Similar expression has been recently determined by Gazetas et al. [19] for dominant rocking systems through a different approach. Furthermore, for the massless rigid foundation (and for the particular case of $c=0$ and $\boldsymbol{p}_{0}=\left[\begin{array}{lll}a_{0} m & 0 & 0\end{array}\right]$ ) Eq. (17) leads to:

$$
\begin{gathered}
a=\frac{a_{0} m\left[H^{2} k\left(\tilde{k}_{e, h}\left(a_{h}\right)\right)+\left(k+\tilde{k}_{e, h}\left(a_{h}\right)\right)\left(\tilde{k}_{e, \theta}\left(a_{\theta}\right)\right)\right]}{H^{2} k^{2} m \omega^{2}-H^{2} k^{2}\left(\tilde{k}_{e, h}\left(a_{h}\right)\right)+\left(H^{2} k+\tilde{k}_{e, \theta}\left(a_{\theta}\right)\right)\left(\left(k+\tilde{k}_{e, h}\left(a_{h}\right)\right)\left(k-m \omega^{2}\right)-k^{2}\right)} \\
a_{h}=\frac{a_{0} k m\left(\tilde{k}_{e, h}\left(a_{h}\right)\right)}{H^{2} k^{2} m \omega^{2}-H^{2} k^{2}\left(\tilde{k}_{e, h}\left(a_{h}\right)\right)+\left(H^{2} k+\tilde{k}_{e, \theta}\left(a_{\theta}\right)\right)\left(\left(k+\tilde{k}_{e, h}\left(a_{h}\right)\right)\left(k-m \omega^{2}\right)-k^{2}\right)} \\
a_{\theta}=\frac{a_{0} H k m\left(\tilde{k}_{e, h}\left(a_{h}\right)\right)}{H^{2} k^{2} m \omega^{2}-H^{2} k^{2}\left(\tilde{k}_{e, h}\left(a_{h}\right)\right)+\left(H^{2} k+\tilde{k}_{e, \theta}\left(a_{\theta}\right)\right)\left(\left(k+\tilde{k}_{e, h}\left(a_{h}\right)\right)\left(k-m \omega^{2}\right)-k^{2}\right)}
\end{gathered}
$$

where

$$
\tilde{k}_{e, h}\left(a_{h}\right)=k_{e, h}\left(a_{h}\right)+i c_{e, h}\left(a_{h}\right) \omega
$$

and 


$$
\tilde{k}_{e, \theta}\left(a_{\theta}\right)=k_{e, \theta}\left(a_{\theta}\right)+i c_{e, \theta}\left(a_{\theta}\right) \omega
$$

are the equivalent complex lateral and rotational stiffnesses, respectively. Also, in Eq. (21) $a$ denotes the steady state amplitude of the top mass. Similar, although lengthier, expressions can be determined by including mass foundation but herein omitted for simplicity's sake.

In order to determine the equivalent period of the nonlinear SSI system it is, therefore, necessary to calculate: i) the equivalent stiffnesses and damping coefficients by applying Eqs (9), (10), (12) and (13); ii) solving the nonlinear Eq. (17) for assigned input frequency $\omega$ to determine the amplitudes $a_{h}$ and $a_{\theta}$; iii) solve the eigenproblem of Eq. (18) to determine the equivalent fundamental periods/frequencies.

It is noted that stiffnesses and damping have been provided in literature for specific cases and soil conditions (see e.g. [19] and [23]) as a function of the foundation displacements and/or rotations. They provide a valuable approximation and can be applied in lieu of equivalent stiffnesses and damping so to avoid step i). In the most general cases, however, the equivalent stiffnesses and damping need to be determined first. In this regard, it is noted, the solution of the integrals given in Eqs. (9), (10), (12), and (13) might be challenging from an analytical point of view and they might need to be solved through numerical integration. In the following sections, the versatile Preisach hysteretic model is tailored for modelling the nonlinear soil-structure interaction and closed form solutions will be provided.

\section{Modelling Nonlinear SSI via the Preisach formalism}

Let assume the nonlinear soil hysteretic elements $f_{i}(x, \dot{x})\left(i=h, \theta\right.$ and $\left.x=u_{F}, \theta\right)$ are given by the superposition of two terms: a zero-memory part $f_{i, Z M}(x, \dot{x})$ and a purely hysteretic part, $f_{i, H}(x, \dot{x})$, monitoring the memory of the system (see e.g. [34], [36]). That is, 


$$
f_{i}(x, \dot{x})=f_{i, Z M}(x)+f_{i, H}(x, \dot{x}) ; i=h, \theta \text { and } x=u_{F}, \theta
$$

The non-hysteretic zero-memory term, $f_{i, Z M}(x)$ in general is nonlinearly related to the instantaneous displacements and rotations only and it is strictly related to the backbone of the nonlinear cyclic soil behaviour. On the other hand, the hysteretic component $f_{i, H}(x, \dot{x})$, monitoring the memory of the system, is a function of the history of $x=u_{F}, \theta$ and it governs the energy dissipation of the system strictly related to the damping mechanism. According to the Preisach formalism, the hysteresis is the result of the superposition of an infinite set of elementary hysteresis operators (hysterons or relay operators) $f_{\alpha, \beta}$, having local memory (Fig. 3), whose explicit expression is given by the following equation (see e.g., $[29,30])$ :

$$
f_{\alpha, \beta}(x, \dot{x})= \begin{cases}+1 & \text { if } x>\alpha \text { or } x>\beta \text { and decreasing } \\ -1 & \text { if } x<\beta \text { or } x<\alpha \text { and increasing }\end{cases}
$$

where $\alpha$ and $\beta$ are the variables that define the Preisach plane.

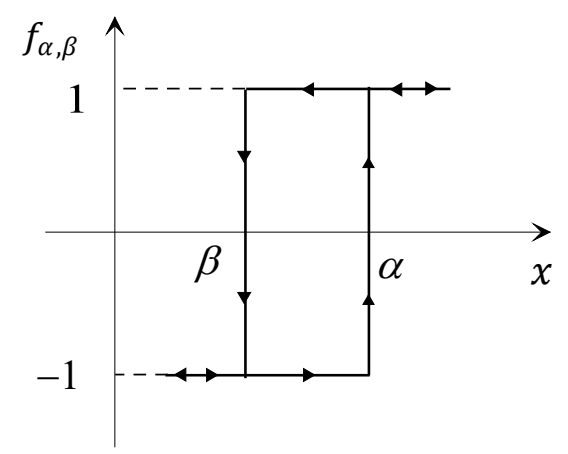

Figure 3: Preisach hysteron

According to Preisach formalism, the hysteretic term $f_{i, H}(x, \dot{x})$ is determined via the equation

$$
f_{i, H}(x, \dot{x})=\iint_{\alpha \geq \beta} \mu(\alpha, \beta) f_{\alpha, \beta}(x, \dot{x}) d \alpha d \beta, \quad i=h, \theta \text { and } x=u_{F}, \theta
$$

where $\mu(\alpha, \beta)$ is an appropriate weight function. Note that, in Eq. (27) the decreasing and the increasing state can be determined either comparing the previous values of the displacement (or the 
rotation) $x(t)$ or through the sign of the velocity $\dot{x}(t)$. In the latter case the velocity assumes the role of events ordering keeping satisfied the rate-independence memory of the hysteresis.

The investigation of the Preisach formalism is considerably facilitated via the geometric interpretation of the model [30]. Indeed, consider now that the weight function $\mu(\alpha, \beta)$ is a finite function defined in the domain $D$ within the equation $\alpha \geq \beta, \alpha=\alpha_{P} ; \beta=\beta_{P}$, defining a triangle in the Preisach plane, as shown in Figure 4. Each point of the domain $D$ in the Preisach plane represents a relay operator. It follows that in a given time instant the domain $D$ can be subdivided in two sub-domains: the domain $S^{+}(x)$ encompassing the set of the relay operators in the +1 status, and the domain $S^{-}(x)$ encompassing the set of the relay operators in the -1 status, see Figure 4 . It can be shown [30], that the interface $L(x)$ is a staircase defined by the dominant maxima and minima of the input $x(t)$.

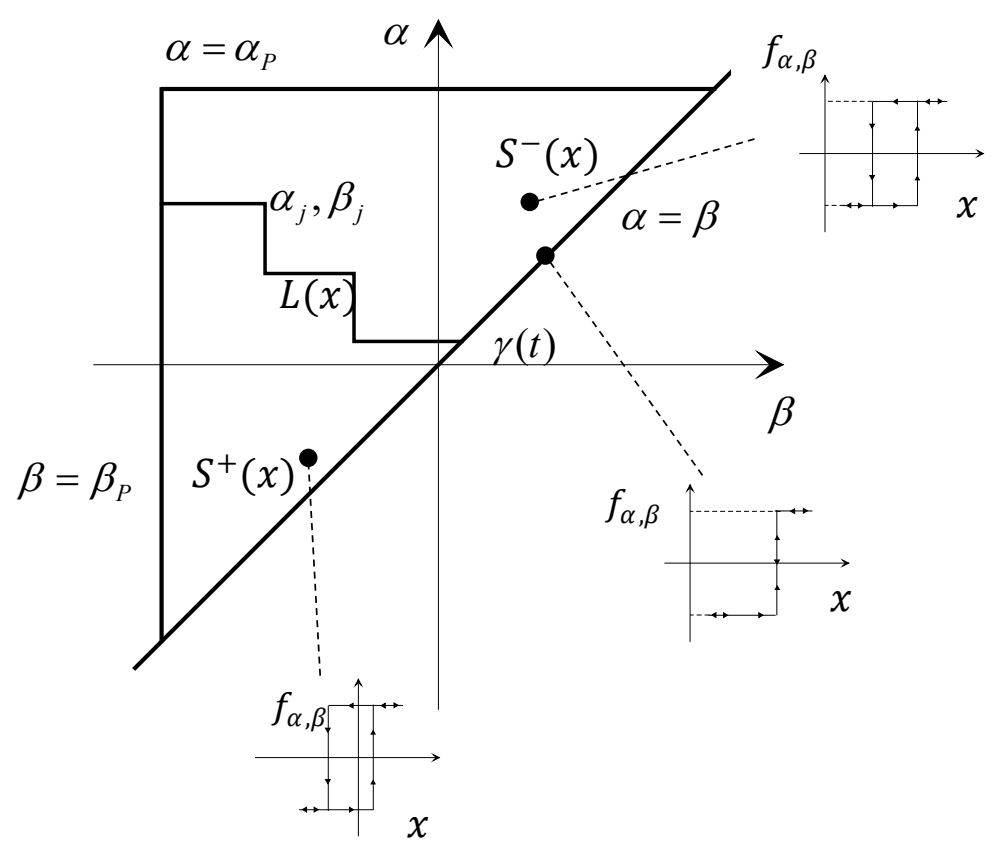

Figure 4: The Preisach Plane

Moreover, if the input increases the final link of the interface will be a horizontal segment, while if the input decreases it will be a vertical segment; both segments originate at a point of the line $\alpha=\beta$. This geometric interpretation allows one to rewrite Eq. (28) in the form 


$$
\begin{array}{r}
f_{i, H}(x, \dot{x})=\iint_{S^{+}(x)} \mu(\alpha, \beta) d \alpha d \beta-\iint_{S^{-}(x)} \mu(\alpha, \beta) d \alpha d \beta=2 \iint_{S^{+}(x)} \mu(\alpha, \beta) d \alpha d \beta-\iint_{D} \mu(\alpha, \beta) d \alpha d \beta \\
i=h, \theta \text { and } x=u_{F}, \theta
\end{array}
$$

Thus, knowing the weight function and the temporal evolution of the domain $S^{+}(x)$ it is possible to evaluate the restoring force $f_{i, H}(x, \dot{x})$. The evolution of the domain $S^{+}(x)$ can be handy determined via its geometric representation. Specifically (see e.g. [30]), the area of the domain $S^{+}(x)$ can be decomposed in a set of triangles bounded by the dominant maxima and dominant minima of $x(t)$. Thus, setting the area of the triangle bounded by the $\mathrm{j}$-th dominant maximum $\alpha_{j}$ and by the $\mathrm{j}$-th dominant minimum $\beta_{j}$ (see Figure 4) equal to

$$
F\left(\alpha_{j}, \beta_{j}\right)=\int_{\beta_{j}}^{\alpha_{j}} \int_{\beta_{j}}^{\alpha} \mu(\alpha, \beta) d \alpha d \beta
$$

the hysteretic term $f_{i, H}(x, \dot{x})$ for the ascending and descending status, Eqs. (28) and (29) are given by the following equations (see e.g. [33], [37]):

$$
f_{i, H}(x, \dot{x})=2\left[F\left(x, \beta_{n-1}\right)+2 F\left(0, \beta_{P}\right)-2 F\left(0, \beta_{0}\right)+\sum_{j=2}^{n-1} F\left(\alpha_{j}, \beta_{j-1}\right)-F\left(\alpha_{j}, \beta_{j}\right)\right]-F\left(\alpha_{P}, \beta_{P}\right)
$$

and

$$
\begin{aligned}
f_{i, H}(x, \dot{x}) & =2\left[F\left(\alpha_{n}, \beta_{n-1}\right)-F\left(\alpha_{n}, x\right)+2 F\left(0, \beta_{P}\right)-2 F\left(0, \beta_{0}\right)+\sum_{j=2}^{n-1} F\left(\alpha_{j}, \beta_{j-1}\right)-F\left(\alpha_{j}, \beta_{j}\right)\right] \\
& -F\left(\alpha_{P}, \beta_{P}\right)
\end{aligned}
$$


Eqs. (31) and (32) can be used along with Eq. (1) to determine the time domain response of the nonlinear SSI system with Preisach hysteresis. Moreover, the equivalent stiffness and damping coefficients can be determined replacing Eqs. (26), (31) and (32) in Eqs. (9), (10), (12) and (13), that is

$$
\begin{aligned}
c_{e, i}\left(a_{i}\right)= & -\frac{1}{\pi \omega a_{i}}\left(\int_{0}^{2 \pi} f_{i, Z M}\left(a_{i} \cos \vartheta\right) \sin \vartheta d \vartheta+\right. \\
& \left.\int_{0}^{2 \pi} f_{i, H}\left(a_{i} \cos \vartheta,-a_{i} \omega \sin \vartheta\right) \sin \vartheta d \vartheta\right)= \\
& -\frac{1}{\pi \omega a_{i}}\left(\int_{0}^{2 \pi} f_{i, Z M}\left(a_{i} \cos \vartheta\right) \sin \vartheta d \vartheta+4 F\left(a_{i},-a_{i}\right)-\right. \\
& \left.2 \int_{0}^{\pi} F\left(a_{i}, a_{i} \cos \vartheta\right) \sin \vartheta d \vartheta+2 \int_{\pi}^{2 \pi} F\left(a_{i} \cos \vartheta,-a_{i}\right) \sin \vartheta d \vartheta\right),
\end{aligned}
$$

and

$$
\begin{aligned}
k_{e, i}\left(a_{i}\right)=\frac{1}{\pi a_{i}} & \left(\int_{0}^{2 \pi} f_{i, Z M}\left(a_{i} \cos \vartheta\right) \cos \vartheta d \vartheta+\int_{0}^{2 \pi} f_{i, H}\left(a_{i} \cos \vartheta,-a_{i} \omega \sin \vartheta\right) \cos \vartheta d \vartheta\right) \\
& =\frac{1}{\pi a_{i}}\left(\int_{0}^{2 \pi} f_{i, Z M}\left(a_{i} \cos \vartheta\right) \cos \vartheta d \vartheta-2 \int_{0}^{\pi} F\left(a_{i}, a_{i} \cos \vartheta\right) \cos \vartheta d \vartheta\right. \\
& \left.+2 \int_{\pi}^{2 \pi} F\left(a_{i} \cos \vartheta,-a_{i}\right) \cos \vartheta d \vartheta\right)
\end{aligned}
$$

In deriving Eqs. (33) and (34), owing to the pseudo-harmonic behavior of the response process, the dominant maxima $\alpha_{j}$ and the dominant minima $\beta_{j}$ have been set equal to the amplitude value and its opposite value respectively. That is:

$$
\alpha_{j}=a_{i} ; \quad \quad \quad \beta_{j}=-a_{i} . \quad i=h, \theta
$$

It has to be emphasized that the weigh function $\mu(\alpha, \beta)$ appearing in Eq. (30) can be determined either experimentally through the first-order transition curves $[30,38]$ or calibrating a model to fit in a least- 
square sense pertinent experimentally or numerically evaluated data (see e.g. [39-40]). Lubarda et al. [38] have derived the weight functions in a closed form for the Jenkins model (i.e. linear spring in series with a Coulomb friction element) [41] and for the Iwan model [26][42], known also as JenkinsIwan distributed model (composed of an infinite number of Jenkins elements). In the following section the particular case of uniform weight function (i.e. Iwan distributed model) will be explored.

\section{Steady-state harmonic response of Preisach nonlinear SSI systems with uniform weight function: Iwan's distributed model}

The distributed Jenkins-Iwan model has been proved to be a particular case of the of the most general Preisach model (see e.g. Lubarda et al. [38], Spanos et al. [33]) In this regard the pertinent weight function $\mu(\alpha, \beta)$, herein tailored for the SSI problem assumes the following form for the horizontal hysteretic element

$$
\mu(\alpha, \beta)=-\frac{k_{h}^{2}}{4} \frac{1}{2 V_{\max }}
$$

and for the rotational element

$$
\mu(\alpha, \beta)=-\frac{k_{\theta}{ }^{2}}{4} \frac{1}{2 M_{\max }}
$$

where $V_{\max }$ and $M_{\max }$ assume the role of the limiting horizontal load and maximum attainable values of overturning moment of the foundation, respectively, while $k_{h}$ and $k_{\theta}$ are the elastic stiffnesses (see e.g. [2], [3]) of the given foundation. Owing to the mathematical structure of the weight functions (36) and (37), after simple algebra, using Eqs. (30), (33) and (34) closed form expressions of the equivalent damping and the equivalent stiffness are determined. Namely, for the horizontal hysteretic element 


$$
c_{e, h}\left(a_{h}\right)=\frac{a_{h} k_{h}^{2}}{3 \pi \omega V_{\max }}
$$

and

$$
k_{e, h}\left(a_{h}\right)=k_{h}-\frac{k_{h}^{2} a_{h}}{4 V_{\max }}
$$

and for the rotational element

$$
c_{e, \theta}\left(a_{\theta}\right)=\frac{a_{\theta} k_{\theta}^{2}}{3 \pi \omega M_{\max }}
$$

and

$$
k_{e, \theta}\left(a_{\theta}\right)=k_{\theta}-\frac{k_{\theta}^{2} a_{\theta}}{4 M_{\max }}
$$

Interestingly, substituting the values of the equivalent damping and stiffnesses in Eqs. (24) and (25) and recalling the definition of "hysteretic" damping (see e.g., Kramer [43]), the loss factors $\eta_{e, h}\left(a_{h}\right)$ and $\eta_{e, \theta}\left(a_{\theta}\right)$, where

$$
\eta_{e, h}\left(a_{h}\right)=\frac{c_{e, h}\left(a_{h}\right) \omega}{k_{e, h}\left(a_{h}\right)}=\frac{4}{12 \pi \frac{V_{\max }}{k_{h} a_{h}}-3 \pi} ; \quad \forall \frac{V_{\max }}{k_{h} a_{h}}<\frac{1}{4}
$$

and

$$
\eta_{e, \theta}\left(a_{\theta}\right)=\frac{c_{e, \theta}\left(a_{\theta}\right) \omega}{k_{e, \theta}\left(a_{\theta}\right)}=\frac{4}{12 \pi \frac{M_{\max }}{k_{\theta} a_{\theta}}-3 \pi} ; \quad \forall \frac{M_{\max }}{k_{\theta} a_{\theta}}<\frac{1}{4}
$$

which are, as expected, amplitude dependent and frequency independent. It has to be emphasized equations (38-41) are determined by the knowledge of the elastic stiffness foundations available in literature (see e.g. [3]) and the limiting horizontal load and maximum attainable values of overturning moment of the foundation that can be determined through a simple pushover analysis or through simplified formulas (see e.g. [19]). Also, it is noted that although Eqs. (33)-(38) can reliably capture 
the main features of cyclic behavior of soil-foundation interaction for low level of the excitation, they might not be able to capture the typical hyperbolic shape of the stiffness backbone and damping.

In the next section a procedure is proposed to calibrate the Preisach model to fully match experimental/numerical evaluated stiffness degradation and damping curves (even beyond the typical inflection point).

\section{Matching stiffness degradation and damping curves}

In this section a procedure for calibrating the Preisach model of hysteresis to fit experimental stiffness degradation and damping curves is proposed. To this aim, by combining Eqs. (26) and (28), the hysteretic element can be rewritten in the following form

$$
f_{i}(x, \dot{x})=f_{i, Z M}(x)+\iint_{\alpha \geq \beta} \mu(\alpha, \beta) f_{\alpha, \beta}(x, \dot{x}) d \alpha d \beta ; i=h, \theta \text { and } x=u_{F}, \theta
$$

Assuming the weigh function $\mu(\alpha, \beta)$ is uniform over the whole domain, that is

$$
\mu(\alpha, \beta)=\chi_{i}
$$

Eq. (30) can be solved in closed form, leading to the following equation

$$
F\left(\alpha_{j}, \beta_{j}\right)=\chi_{i} \frac{1}{2}\left(\beta_{j}-\alpha_{j}\right)^{2}
$$

The equivalent stiffness given in Eq. (34), that is set coincident with the experimentally or numerically evaluated stiffness degradation, reduces to

$$
k_{e, i}\left(a_{i}\right)=\frac{1}{\pi a_{i}}\left(\int_{0}^{2 \pi} f_{i, Z M}\left(a_{i} \cos \vartheta\right) \cos \vartheta d \vartheta\right)+2 \chi_{i} a_{i} \equiv k_{b b, i}\left(a_{i}\right)
$$

It can be easily proved that Eq. (42) is identically satisfied for the following zero-memory counterpart of the hysteretic element:

$$
f_{i, Z M}(x)=k_{b b, i}\left(a_{i}\right) x-2 \chi_{i} a_{i} x
$$


The damping coefficient, on the other hand, given by Eq. (33) reduces to

$$
c_{e, i}\left(a_{i}\right)=-\frac{8 a_{i} \chi_{i}}{3 \pi \omega}
$$

and rearranging the above equation, the unknown value of the weight function $\mu(\alpha, \beta)=\chi_{i}$ is retrieved imposing the matching with the damping curve $c_{\text {exp }, i}\left(a_{i}\right)$ experimentally or numerically evaluated (see e.g. Li et al. [23] and Kramer [43])

$$
\chi_{i}=-\frac{3 \pi \omega c_{\text {exp }, i}\left(a_{i}\right)}{8 a_{i}} \quad a_{i}>0
$$

Finally, the Preisach (or also Preisach-Iwan) model of hysteresis matching given stiffness degradation and damping curves is given in compact form as

$$
f_{i}(x, \dot{x})=k_{b b, i}\left(a_{i}\right) x+\chi_{i}\left(\iint_{\alpha \geq \beta} f_{\alpha, \beta}(x, \dot{x}) d \alpha d \beta-2 a_{i} x\right) ; i=h, \theta \text { and } x=u_{F}, \theta
$$

in which the first term represents the backbone of the hysteresis loop while the second term monitors the memory of the system and the energy dissipation purged by the $2 a_{i} x$ term so to match a given stiffness degradation curve. It has to be emphasized that as both modulus reduction and damping curves are present in the calibration process, the proposed model offers a promising strategy to overcome the persistent problem with the more widely used non-linear soil models to match simultaneously modulus reduction and damping curves.

\section{$7 \quad$ Numerical Results}

\subsection{Harmonic steady state response of nonlinear Preisach-Iwan rocking dominated and translational dominated systems}


In this section the harmonic steady state response of two classical models used to investigate the key features of soil-structure-interaction systems is explored. Namely, massless foundation translational dominated and rocking dominated systems depicted in Figures 5 are herein discussed.

a)

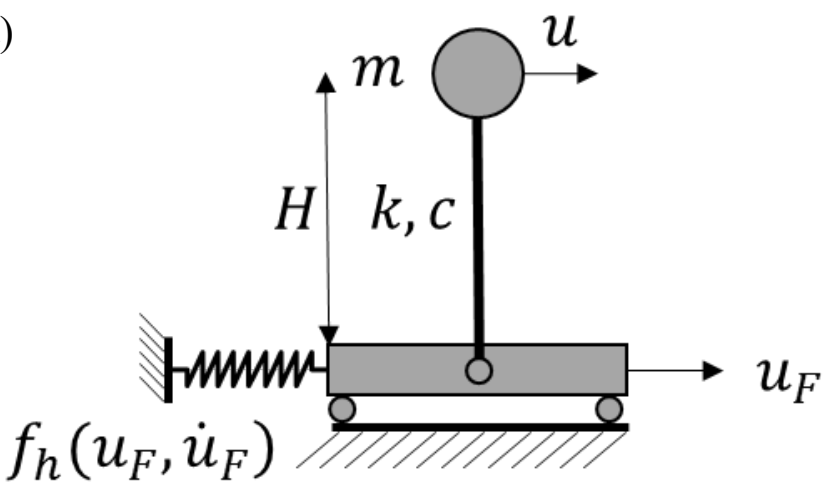

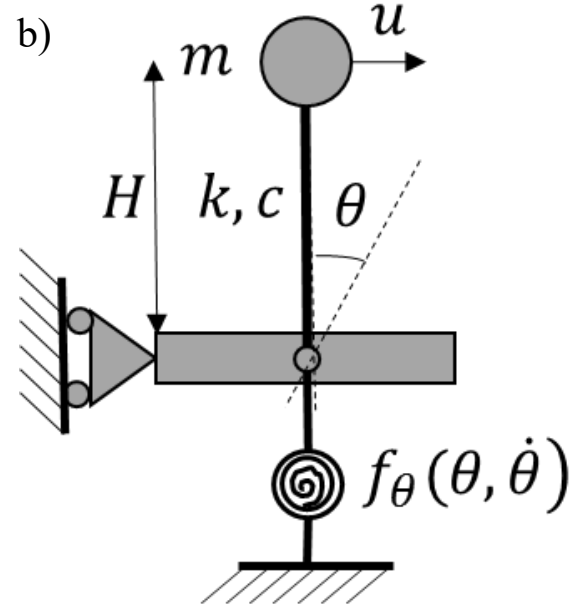

Figure 5: 2-DoFs nonlinear soil structure systems: a) translational dominated; b) rocking dominated

By adopting the proposed equivalent spring and damping closed form expressions determined in section 5 through the Preisach-Iwan formulation, the steady state solution for the translational dominated system Fig 5a are given substituting Eqs. (38) and (39) into Eq. (17)

$$
\begin{gathered}
a=\frac{a_{0} m\left[a_{h} k_{h}^{2}(4 i-3 \pi)+12\left(k+k_{h}\right) \pi V_{\max }\right]}{-a_{h} k_{h}^{2}(-4 i+3 \pi)\left(k-m \omega^{2}\right)+12 \pi V_{\max }\left(k k_{h}-\left(k+k_{h}\right) m \omega^{2}\right)} \\
a_{h}=-\frac{12 a_{0} m k \pi V_{\max }}{a_{h} k_{h}^{2}(-4 i+3 \pi)\left(k-m \omega^{2}\right)+12 \pi V_{\max }\left(k k_{h}-\left(k+k_{h}\right) m \omega^{2}\right)}
\end{gathered}
$$

and for the rocking dominated system (Figure 5b) by substituting Eqs. (40) and (41) into Eq. (17)

$$
\begin{gathered}
a=\frac{a_{0} m\left[a_{\theta} k_{\theta}^{2}(-4 i+3 \pi)-12\left(H^{2} k+k_{\theta}\right) \pi M_{\max }\right]}{a_{\theta} k_{\theta}^{2}(-4 i+3 \pi)\left(k-m \omega^{2}\right)+12 \pi M_{\max }\left(-k k_{\theta}-\left(H^{2} k+k_{\theta}\right) m \omega^{2}\right)} \\
a_{\theta}=-\frac{12 \pi a_{0} m H k M_{\max }}{a_{\theta} k_{\theta}^{2}(-4 i+3 \pi)\left(k-m \omega^{2}\right)+12 \pi M_{\max }\left(-k k_{\theta}-\left(H^{2} k+k_{\theta}\right) m \omega^{2}\right)}
\end{gathered}
$$


Representing nonlinear algebraic expression that can be readily solved through traditional numerical procedures. Interestingly, by introducing the following set of non-dimensional parameters in Eqs. (52) and (53) for the translational dominated system:

$$
\psi=\frac{a_{0} m}{V_{\max }} ; \quad Q=\frac{a_{h} k_{h}}{V_{\max }} ; \quad Q_{a}=\frac{a k}{V_{\max }} ; \quad \alpha=\frac{\omega_{0}}{\omega_{h}} ; \quad \eta=\frac{\omega}{\omega_{h}}
$$

and for the rocking dominated system in Eqs. (54) and (55)

$$
\psi=\frac{a_{0} m H}{M_{\max }} ; \quad Q=\frac{a_{\theta} k_{\theta}}{M_{\max }} ; \quad Q_{a}=\frac{a k H}{M_{\max }} ; \quad \alpha=\frac{\omega_{0}}{\omega_{\theta}} ; \quad \eta=\frac{\omega}{\omega_{\theta}}
$$

and taking the absolute value, it is possible to cast a single nondimensional steady state solution for both translational dominated and rotational dominated systems, that is:

$$
Q_{a}=\alpha^{2} \psi \sqrt{\frac{16 Q^{2}+9 \pi^{2}\left(Q-4 \alpha^{2}\right)^{2}}{16 Q^{2}\left(\alpha^{2}-\eta^{2}\right)^{2}+9 \pi^{2}\left(Q \eta^{2}-\alpha^{2}\left(-4+Q+4 \eta^{2}\right)\right)^{2}}}
$$

and

$$
Q=\frac{12 \pi \alpha^{2} \psi}{\sqrt{16 Q^{2}\left(\alpha^{2}-\eta^{2}\right)^{2}+9 \pi^{2}\left(Q \eta^{2}-\alpha^{2}\left(-4+Q+4 \eta^{2}\right)\right)^{2}}}
$$

where $Q_{a}$ is the non-dimensional amplitude of the top displacement and $Q$ is the nondimensional amplitude of the base motion (i.e the base translation or rotation). Figures (6) and (7) depict Eqs. (58) and (59) for a set of selected parameters and for fixed values of the parameter $\alpha$ (i.e. the ratio between the fully fixed frequency and the SSI fundamental frequency) and compared with the results determined from the time domain analysis, i.e. solving eq. (1) along with Eqs. (31) and (32) thought the $4^{\text {th }}$ order Runge-Kutta scheme. 

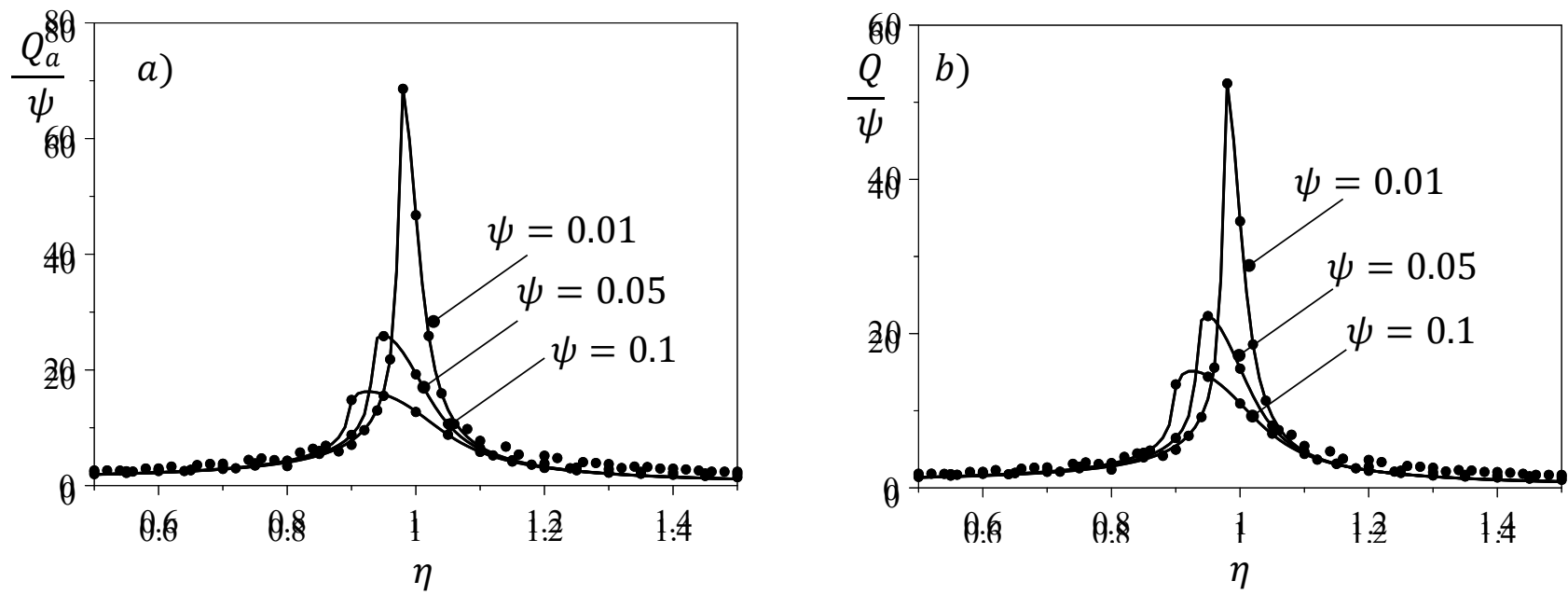

Figure 6 Non-dimensional steady state harmonic response for translational and rocking dominated nonlinear

SSI systems for various values of the normalized amplitude $\psi$ and frequencies ratio $\alpha=1.2$ a) top displacement and b) base translation and/or rotation. Proposed approach (solid line) and time history analysis (symbols)
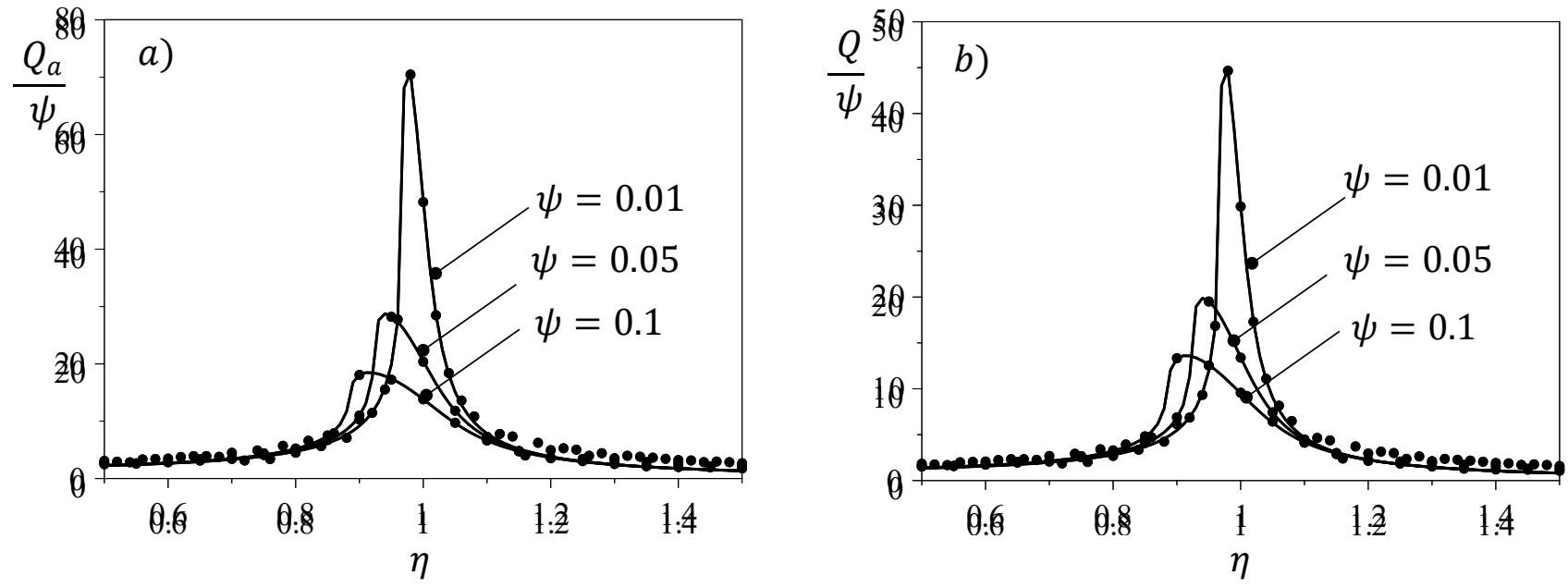

Figure 7 Non-dimensional steady state harmonic response for translational and rocking dominated nonlinear

SSI systems for various values of the normalized amplitude $\psi$ and frequencies ratio $\alpha=1.3$ a) top displacement and b) base translation and/or rotation. Proposed approach (solid line) and time history analysis (symbols) 
From the exam of the figures, it can be seen the excellent matching of the proposed approximated solution of the harmonic steady state responses and the numerical integration. It has to be emphasized the numerical integration required an ad-hoc implementation. Even if the Runge Kutta procedure is embedded in various mathematical packages, the solution of Eq. (1) in conjunction with Eqs. (31) and (32) requires the storage of the dominant maxima and dominant minima at each step, which is difficult to combine with built-in subroutines.

Moreover, the figures show clearly the range of values in which nonlinear SSI provides larger values respect to the linear assumption of the SSI despite there is an increment of the damping ratio and a period elongation. The latter can be determined by introducing the non-dimensional parameters given in Eqs. (56) and (57). Specifically, the stiffness degradation and the equivalent loss factor are given by the following equations

$$
\frac{k_{e, i}(Q)}{k_{i}}=1-\frac{Q}{4} ; \quad \forall Q<4 ; i=h, \theta
$$

and

$$
\eta_{e, i}(Q)=\frac{4 Q}{12 \pi-3 \pi Q} ; \quad \forall Q<4 ; i=h, \theta
$$

while the ratio of the equivalent period over the fully fixed period is given by

$$
\frac{T_{e}(Q)}{T}=\sqrt{\frac{\varphi}{1-\frac{Q}{4}}+1}
$$

where the non-dimensional parameter $\varphi$ is introduced and it assumes the following values for soilstructure-interaction systems translational dominated

$$
\varphi=\frac{k}{k_{h}}
$$

and for rotational dominated 


$$
\varphi=\frac{k H^{2}}{k_{\theta}}
$$
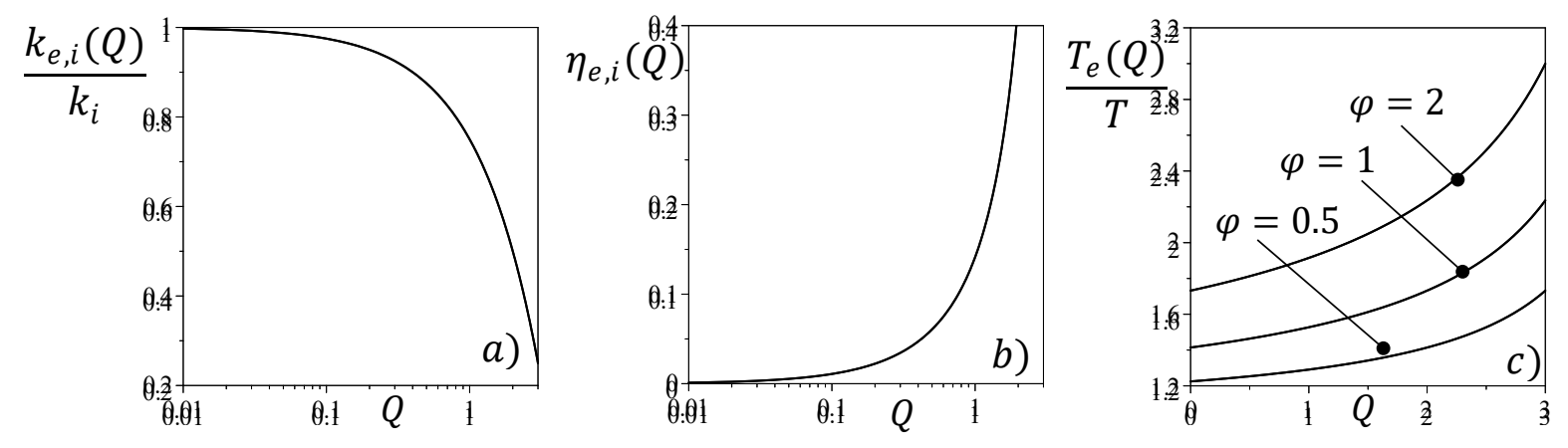

Figure 8 Stiffness degradation a) loss factor b) and ratio of equivalent period c) versus the non-dimensional base displacement (or rotation) amplitude.

From the exam of Figure 8, it is clear that the Preisach-Iwan model is able to capture typical stiffness degradation and damping curve up to the inflection point.

\subsection{Harmonic steady state response of a simple structure on batter pile foundation}

In this section, the steady state response of a simple structure on batter pile foundation resting on Fontainebleau sand extensively studied by $\mathrm{Li}$ et al [23] and validated by centrifuge tests is herein addressed. The nonlinear soil-structure interaction problem is modelled by a 3DoF system (see Figure

2) with base horizontal and rotational spring and dashpot elements given by the following equations [23]:

$$
\begin{gathered}
k_{h}\left(a_{h}\right)=\frac{k_{h \max }}{1+\alpha \mathrm{h} a_{h}{ }^{\beta h}} \\
c_{h}\left(a_{h}\right)=\operatorname{Dhmax}\left(m_{h}\left(\frac{k_{h}\left(a_{h}\right)}{k_{h \max }}\right)^{2}-n_{h}\left(\frac{k_{h}\left(a_{h}\right)}{k_{h \max }}\right)+1\right) 2 \sqrt{k_{h}\left(a_{h}\right) \mathrm{m}_{F}}
\end{gathered}
$$




$$
\begin{gathered}
k_{\theta}\left(a_{\theta}\right)=\frac{k_{\theta \max }}{1+\alpha \theta a_{\theta}{ }^{\beta \theta}} \\
c_{\theta}\left(a_{\theta}\right)=\mathrm{D} \theta \max \left(m_{\theta}\left(\frac{k_{\theta}\left(a_{\theta}\right)}{k_{\theta \max }}\right)^{1.1}-n_{\theta}\left(\frac{k_{\theta}\left(a_{\theta}\right)}{k_{\theta \max }}\right)+1\right) 2 \sqrt{k_{\theta}\left(a_{\theta}\right) \mathrm{I}_{\mathrm{F}}} ;
\end{gathered}
$$

whose relevant parameters are given in Table 1.

Table 1: Main constant for horizontal and rotational stiffness degradation and damping curves for batter pile foundation after Li et al. [23]

\begin{tabular}{lllllll}
$\mathrm{i}=h, \theta$ & \multicolumn{1}{c}{$k_{\text {imax }}$} & $\alpha \mathrm{i}$ & $\beta \mathrm{i}$ & Dimax - & $m_{i}$ & $n_{i}$ \\
& & & & & & \\
\hline Horizontal & $2.3 \times 10^{5} \mathrm{kN} / \mathrm{m}$ & 200 & 1.05 & 0.6 & 0.88 & 1.8 \\
& & & & & & \\
Rotational & $2.35 \times 10^{6} \mathrm{kNm} / \mathrm{rads}$ & $5.0 \times 10^{3}$ & 1.4 & 0.6 & 0.63 & 1.5
\end{tabular}

Additional details can be found in the pertinent reference [23]. The lateral stiffness degradation and damping curves are depicted in Figures 9 and 10. Specifically, Figure 9 shows the lateral stiffness and damping versus the base displacements

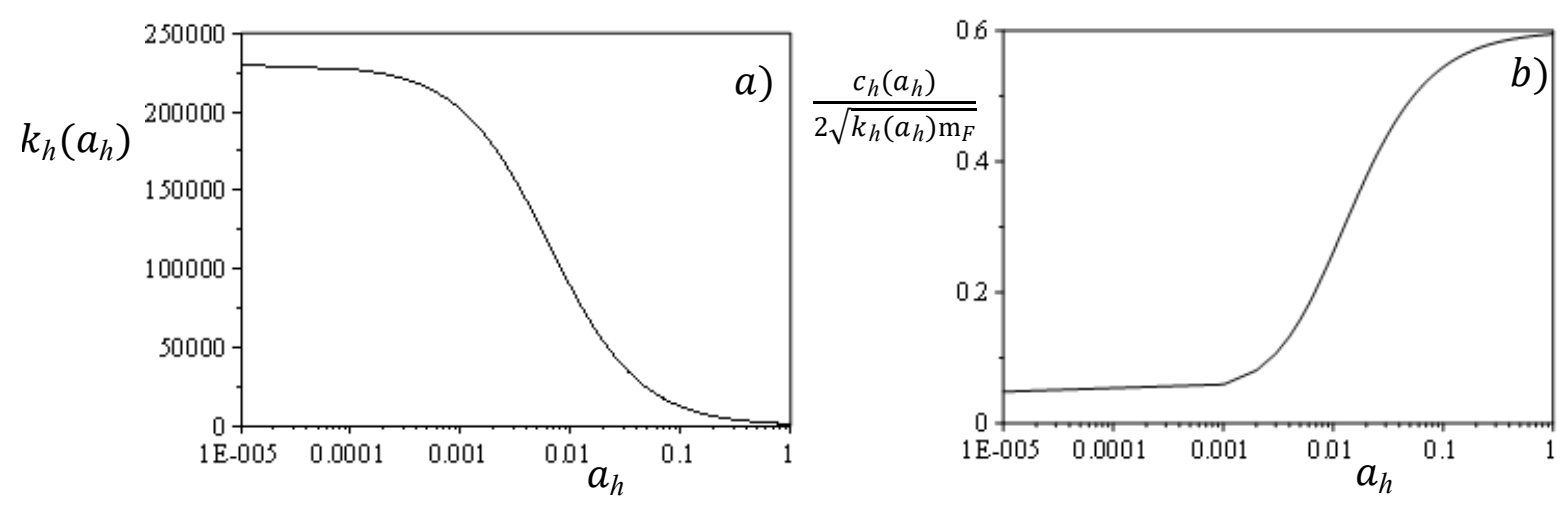

Figure 9 Horizontal stiffness degradation a) and damping b) curves for batter pile foundation after Li et al [23] 
while the degradation of the rotational stiffness and damping are depicted in Figure 10

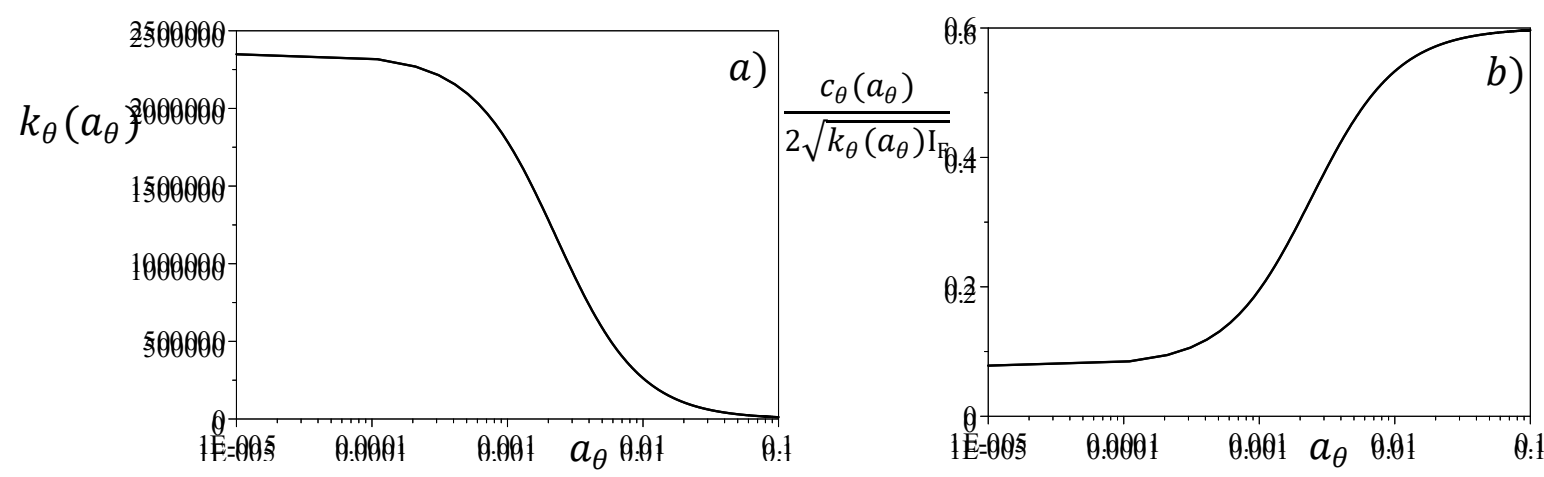

Figure 10 Rotational stiffness degradation a) and damping b) curves for batter pile foundation after Li et al [23]

The procedure proposed in section 6 is therefore herein applied. The steady state response is then evaluated and compared with the results from the time history analyses. Relevant results are reported in Figures 11 and 12. Excellent match is determined also in this case despite the strong nonlinearity. Moreover, Figures 13 and 14 present the comparisons of the numerically evaluated trajectories (harmonic input at $2 \mathrm{~Hz}$ ) of the absolute top mass displacements $\left(u_{T}=u+u_{F}+H \theta\right)$ for both superstructure heights $\mathrm{H}=5.12 \mathrm{~m}$ and $\mathrm{H}=14.16 \mathrm{~m}$ with the steady state solution determined through the proposed approach. Steady state results are in excellent agreement and practically coincident with the centrifuge results provided in [23]. 

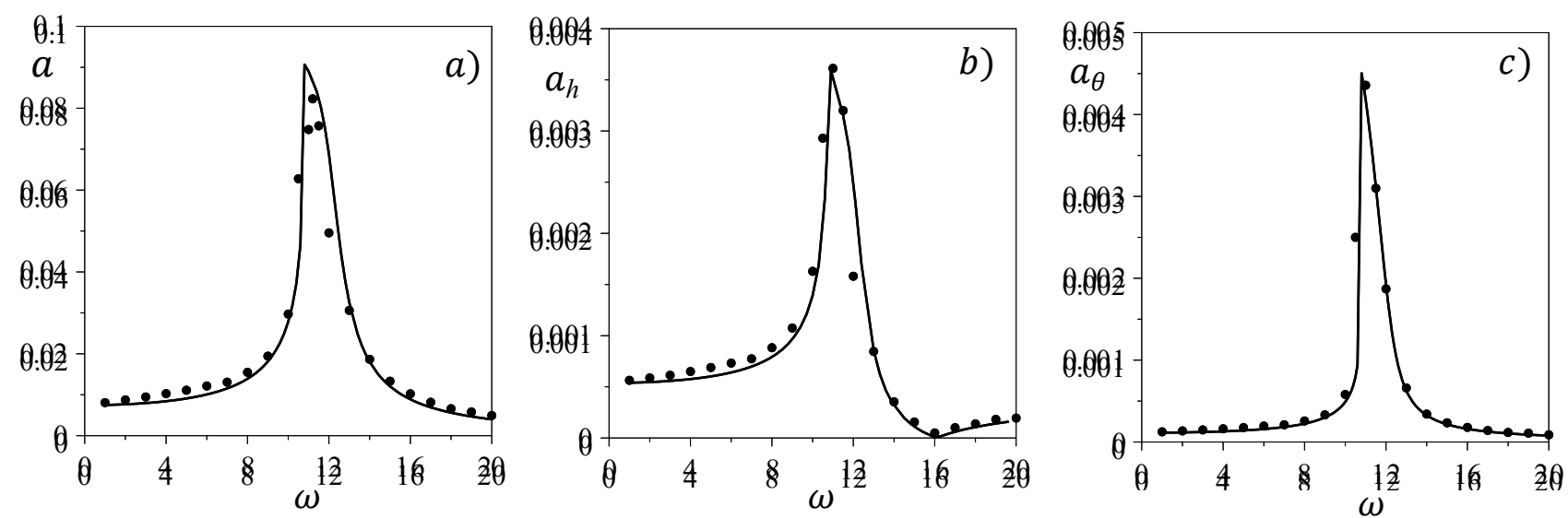

Figure 11 Steady state response of the 3Dof System after Li et al [23] for batter pile foundation and $\mathrm{H}=5.12 \mathrm{~m}$ : a) top relative displacement, b) base translation c) base rotation. Proposed approach (solid line) and time history analysis (symbols)
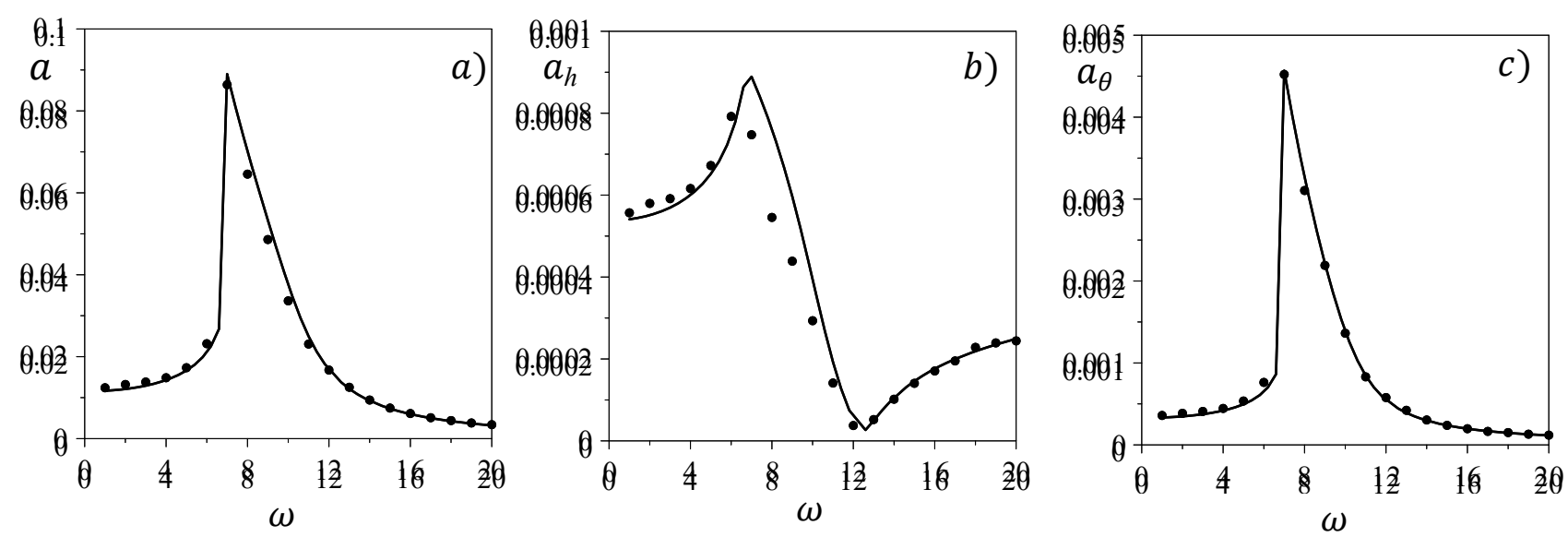

Figure 12 Steady state response of the 3Dof System after Li et al [23] for batter pile foundation and $\mathrm{H}=14.16 \mathrm{~m}$ : a) top relative displacement, b) base translation c) base rotation. Proposed approach (solid line) and time history analysis (symbols) 


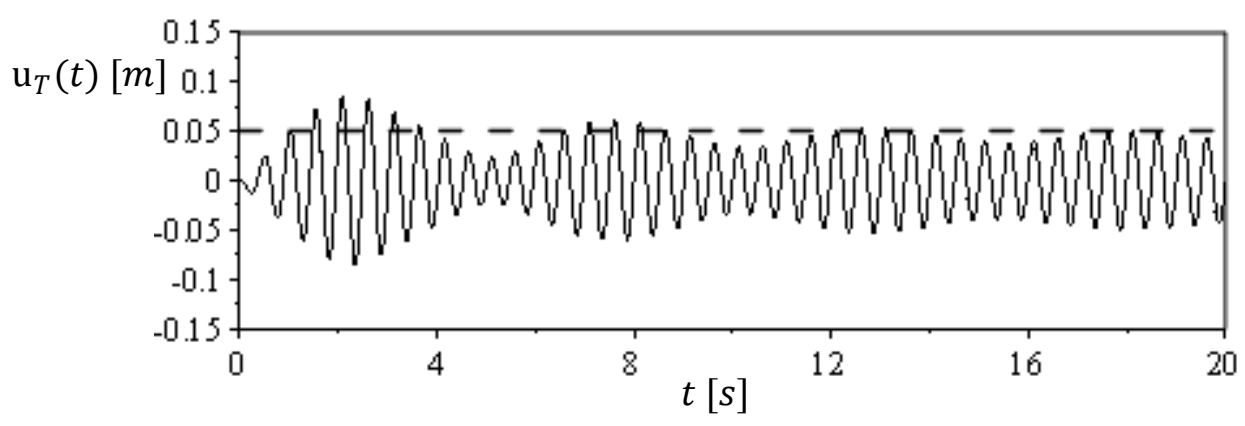

Figure 13 Comparison of the time history of the absolute top mass displacement (solid line) and steady state solution (dashed line) after $\mathrm{Li}$ et al [23] for batter pile foundation and $\mathrm{H}=5.12 \mathrm{~m}$

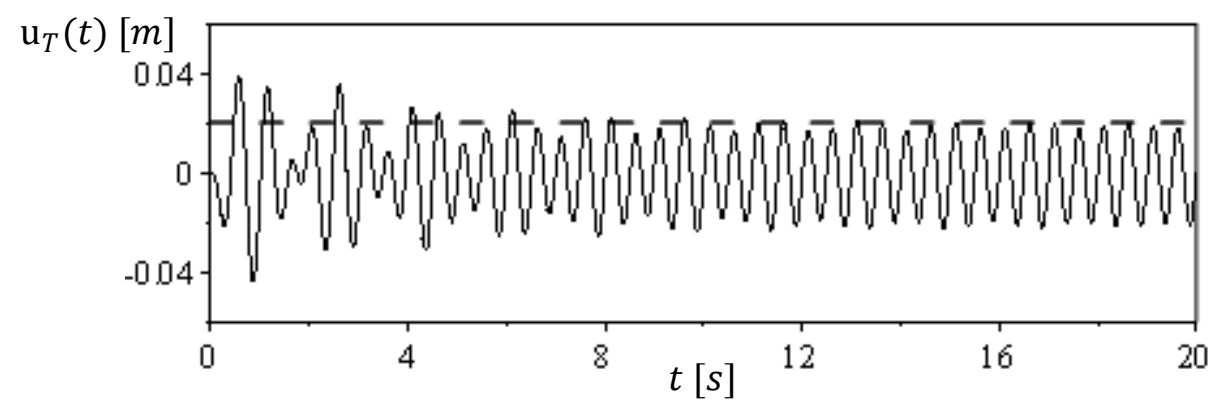

Figure 14 Comparison of the time history of the absolute top mass displacement (solid line) and steady state solution (dashed line) after $\mathrm{Li}$ et al [23] for batter pile foundation and $\mathrm{H}=14.16 \mathrm{~m}$

\subsection{Harmonic steady state response of an onshore wind turbine on a shallow foundation}

In this section a practical application of the proposed approach is presented to analyse the steady state response of the 1.5 MW reference wind turbine designed by the National Renewable Energy Laboratory [44-45] reported in Figure 15 . The wind turbine tower is $82.3 \mathrm{~m}$ tall supported by a $17 \mathrm{~m}-$ diameter circular foundation. The marine soil deposit is a saturated Drammen clay [46] with a plasticity index PI $=27 \%$, a clay content of $45-55 \%$ and Liquid Limit of 55 . The upper soil layer is a 25.5m-thick soft clay over a 42.5m-thick medium-hard clay layer; properties are reported in Table 2 . The finite element model of the whole soil-foundation-structure system is depicted in Fig 16 . The 
steel tower is simplified through beam elements with a constant hollow cross-section of diameter 4.6m and thickness $0.013 \mathrm{~m}$. An elastic material with elastic modulus, $E$, of $2.0 \times 10^{11} \mathrm{~Pa}$ and Poisson's coefficient, $v$, of 0.3 , is used. The total mass of the tower and of its components is idealized as lumped mass, $\mathrm{m}_{\mathrm{wt}}$, of $78600 \mathrm{~kg}$ at the top of the tower. The disk foundation of $3 \mathrm{~m}$ thickness is modelled as rigid body with mass of $1634257 \mathrm{~kg}$ and moment of inertia of $33195835 \mathrm{kgm}^{2}$.

The upper soil layer is simulated as ideal elastic-plastic material with yield stress equal to the undrained cohesion of Table 2 whilst the bottom layer is considered to remain elastic.

The nonlinear soil-structure interaction problem is therefore addressed through the Preisach-Iwan model using the closed form expressions presented in Eqs. (38)-(41) with elastic translational and rotational stiffness given by the following expressions [47]:

$$
\begin{gathered}
k_{h}=\frac{8 G_{S}^{1} R}{2-v_{S}^{1}} \frac{1+\frac{R}{2 H_{S}}}{1+\frac{R G_{S}^{1}}{2 H_{S} G_{S}^{2}}} \\
k_{\theta}=\frac{8 G_{s}^{1} R^{3}}{3\left(1-v_{S}^{1}\right)} \frac{1+\frac{R}{6 H_{S}}}{1+\frac{R G_{S}^{1}}{6 H_{S} G_{S}^{2}}}
\end{gathered}
$$

where $G_{S}^{i}$ is the shear modulus of the ith-layer, $\mathrm{R}$ is the radius of the foundation and $H_{S}$ is the thickness of the upper layer. Using the data reported in Table 2, the calculated soil-foundation translational and rotational stiffness are equal to $k_{h}=9.856 \times 10^{8} \mathrm{~N} / \mathrm{m}, \quad k_{\theta}=5.3585 \times 10^{10} \mathrm{Nm} / \mathrm{rad}$ respectively.

A pushover analysis is performed to derive the ultimate translational and rotational capacity of the foundation, $V \max =4657790 \mathrm{~N}$ and $\mathrm{Mmax}=50146770 \mathrm{Nm}$, respectively. The latter values along with Eqs. (69) and (70) are used in Eqs (38-41) to define the equivalent stiffness and damping according to the Preisach-Iwan model. 
Nonlinear analyses are carried out by considering an external harmonic forces at several frequencies applied at the top of the tower with intensity $F_{0}=0.5 m_{w t}$ and $F_{0}=1.0 m_{w t}$. A dynamic implicit step-by-step algorithm is used to perform the dynamic analysis in ADINA by setting the Newmark's parameters, $\gamma=0.6$ and $\beta=0.3025$, to damp out noises and to stabilize the integration scheme (see e.g. [48]).

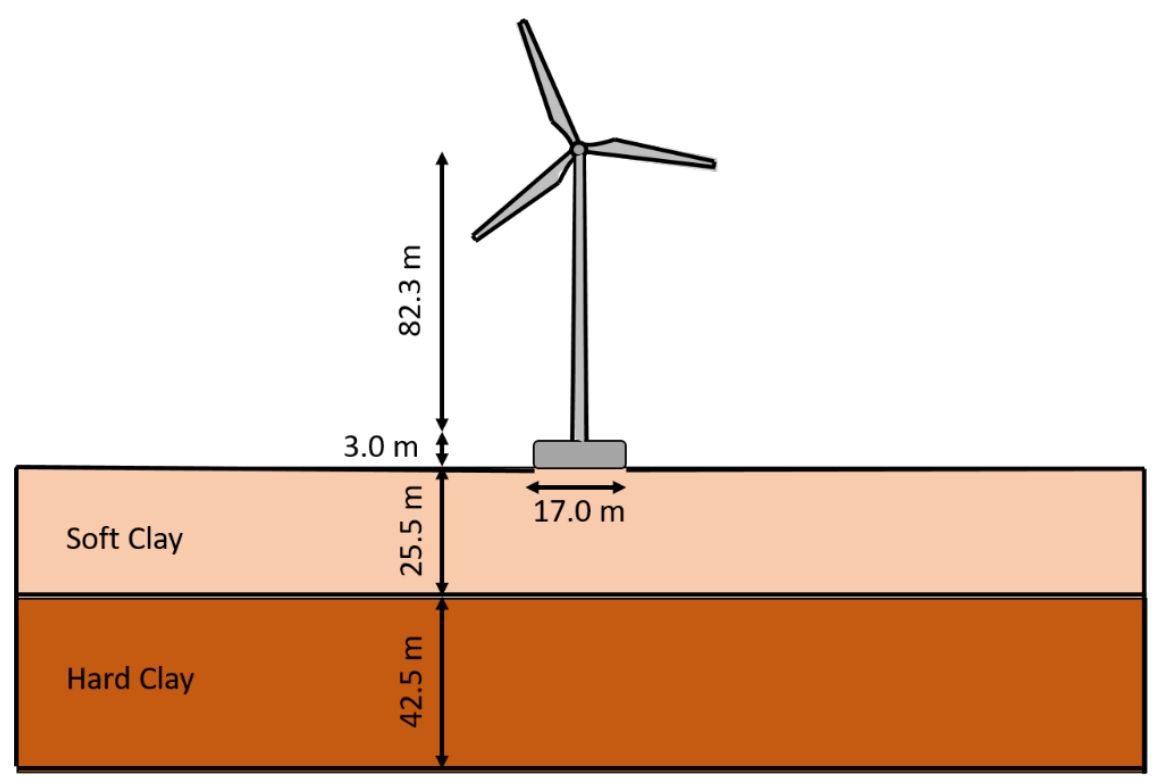

Figure 15: Geometry of the wind turbine and soil stratum for the nonlinear SSI analysis 


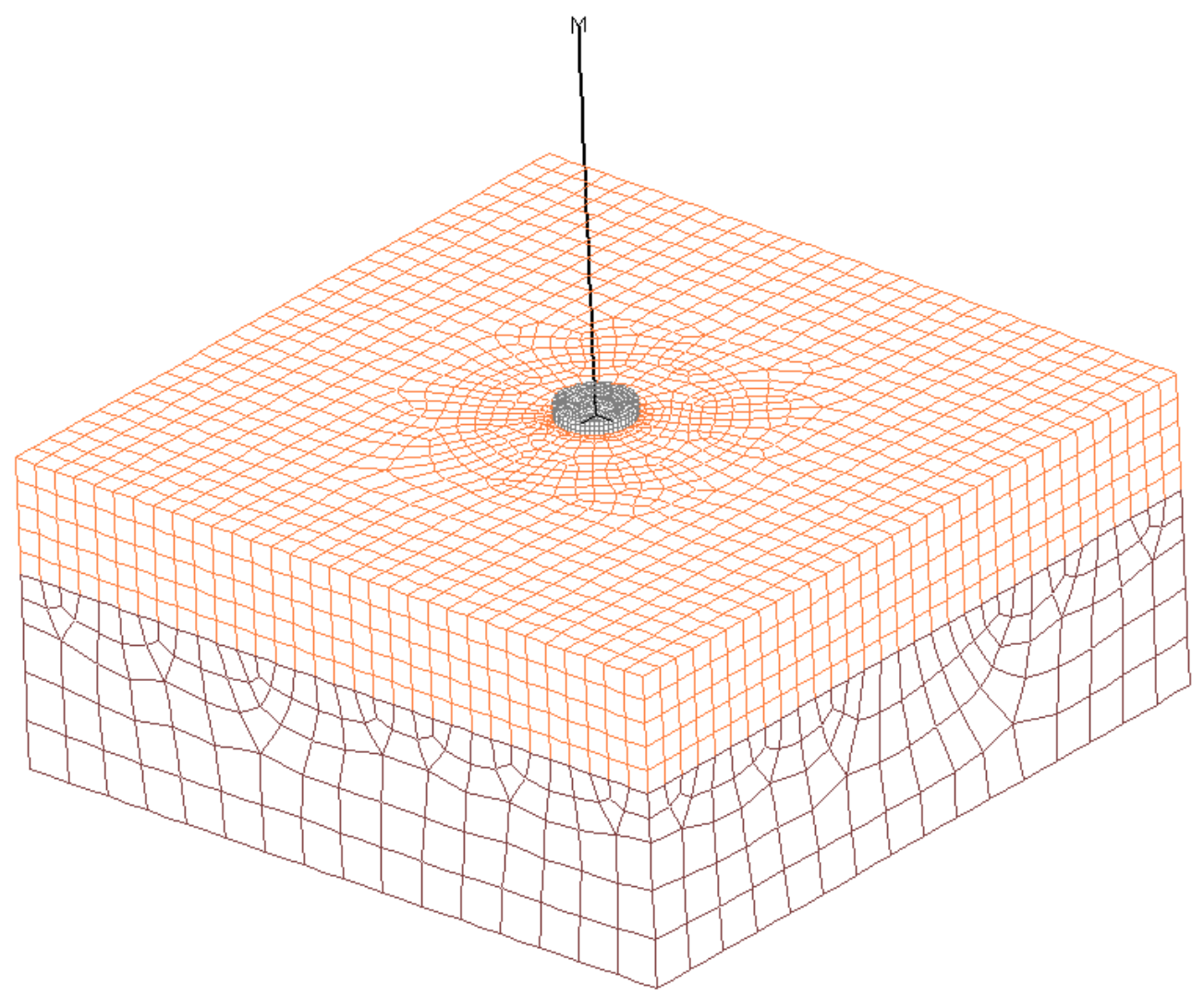

Figure 16: FE model of the wind turbine and soil stratum for the nonlinear SSI analysis

Table 2: Geometrical and mechanical soil data

\begin{tabular}{ccccccc} 
Soil & Vs & $\mathrm{H}_{\mathrm{s}}[\mathrm{m}]$ & $G_{s}[\mathrm{~Pa}]$ & $v_{s}$ & $\rho_{s}\left[\mathrm{~g} / \mathrm{m}^{3}\right]$ & $C_{u}[\mathrm{~Pa}]$ \\
\hline Soft Clay & 100 & 25.5 & 22000000 & 0.3 & 2200 & 30000 \\
Hard Clay & 200 & 42.5 & 88000000 & 0.3 & 2200 & -
\end{tabular}

Figure 17 shows the comparison of the steady state calculated through the proposed approach modelling the wind turbine as 3Dof system and through the time history analysis performed in ADINA. Excellent match has been found also in this case. It is worth to emphasize from a computational point of view that the whole curve determined through the proposed approach required few seconds to be run while the FE solution required few hours. 


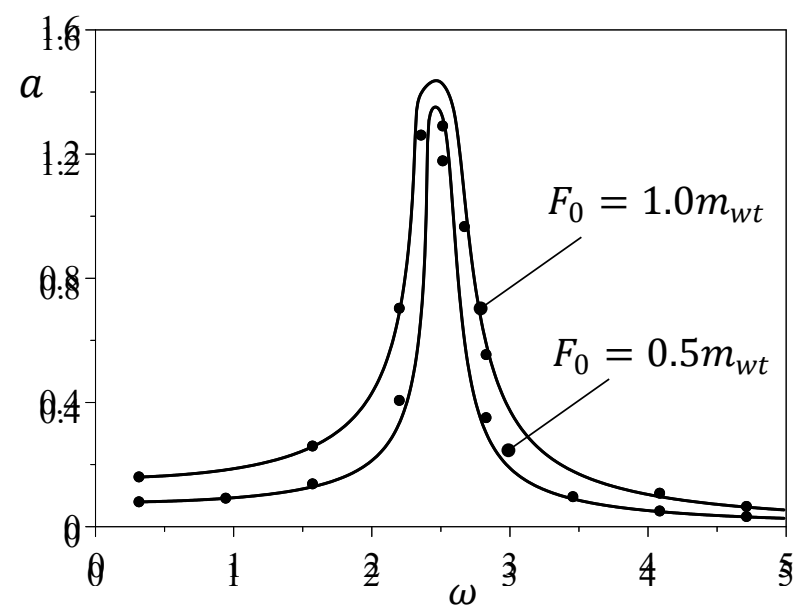

Figure 17: Steady state response of the top displacements of the wind turbine: comparison between the proposed method (solid line) and FE results (symbols).

\section{Concluding Remarks}

In this paper, the steady state response for nonlinear soil-structure interaction problems is determined. To the best knowledge of the authors this is the first contribution addressing the steady state study of nonlinear soil-structure interaction problems in analytical (although simplified) fashion. By modelling the soil through a set of horizontal and rotational nonlinear springs, the harmonic balance approach is applied. Various closed form solutions have been derived to determine the equivalent period, damping and steady state amplitude. Furthermore, the Preisach formalism has been adopted to capture the hysteretic behaviour of soil for small and medium strain condition (therefore, the dependency of stiffness and damping from the number of cycles has been neglected). It has to be pointed out that that is not a limitation of the Preisach formalism as it might be able to capture very complicated phenomena including strain degradation for large deformations and foundation uplifting, but for those cases the dynamic response might not exhibit a steady state, therefore those phenomena have not been included in this paper. By using the Preisach-Iwan type hysteretic model, the proposed approach led to closed form solutions of the equivalent stiffness and damping foundation as a function of the ultimate base horizontal force and moment and the elastic foundation stiffnesses. Despite the 
simplicity of the soil-structure-interaction model the comparison with realistic case scenarios showed an excellent accuracy and therefore the potential use for predesign purposes in a wide range of applications.

\section{References}

[1]E. Kausel, "Early history of soil-structure interaction," Soil Dynamics and Earthquake Engineering, vol. 30, no. 9, pp. 822-832, Sep. 2010, doi: 10.1016/j.soildyn.2009.11.001. [2]H. G. Poulos and E. H. Davis, Elastic solutions for soil and rock mechanics. [New York: Wiley, 1973.

[3]R. Dobry and G. Gazetas, "Dynamic Response of Arbitrarily Shaped Foundations," Journal of Geotechnical Engineering, vol. 112, no. 2, pp. 109-135, Feb. 1986, doi: 10.1061/(ASCE)07339410(1986)112:2(109).

[4]C. Bolisetti, A. S. Whittaker, and J. L. Coleman, "Linear and nonlinear soil-structure interaction analysis of buildings and safety-related nuclear structures," Soil Dynamics and Earthquake Engineering, vol. 107, pp. 218-233, Apr. 2018, doi: 10.1016/j.soildyn.2018.01.026.

[5]M. Ciampoli and P. E. Pinto, "Effects of Soil-Structure Interaction on Inelastic Seismic Response of Bridge Piers," Journal of Structural Engineering, vol. 121, no. 5, pp. 806-814, May 1995, doi: 10.1061/(ASCE)0733-9445(1995)121:5(806).

[6]M. Jakub and J. M. Roesset, "Nonlinear Stiffness of Foundations.," National Science Foundation, Washington, DC, MIT-CE-R77-35, 1977. [Online]. Available:

https://nehrpsearch.nist.gov/article/PB-286\%20653/1/XAB.

[7]M. P. Santisi d'Avila and F. Lopez-Caballero, "Analysis of nonlinear soil-structure interaction effects: 3D frame structure and 1-Directional propagation of a 3-Component seismic wave," Computers \& Structures, vol. 207, pp. 83-94, Sep. 2018, doi: 10.1016/j.compstruc.2018.02.002. [8]A. Pecker and C. T. Chatzigogos, "Non linear soil structure interaction: impact on the seismic response of structures," presented at the XIV European Conference on Earthquake Engineering, Ohrid, FYROM, 2010.

[9]C. T. Chatzigogos, R. Figini, A. Pecker, and J. Salençon, "A macroelement formulation for shallow foundations on cohesive and frictional soils," Int. J. Numer. Anal. Meth. Geomech., vol. 35, no. 8, pp. 902-931, Jun. 2011, doi: 10.1002/nag.934.

[10]R. Figini, R. Paolucci, and C. T. Chatzigogos, “A macro-element model for non-linear soilshallow foundation-structure interaction under seismic loads: theoretical development and experimental validation on large scale tests: A MACRO-ELEMENT FOR NON-LINEAR SOILFOUNDATION-STRUCTURE INTERACTION," Earthquake Engng Struct. Dyn., vol. 41, no. 3, pp. 475-493, Mar. 2012, doi: 10.1002/eqe.1140.

[11]F. Cavalieri, A. A. Correia, H. Crowley, and R. Pinho, "Dynamic soil-structure interaction models for fragility characterisation of buildings with shallow foundations," Soil Dynamics and Earthquake Engineering, vol. 132, p. 106004, May 2020, doi: 10.1016/j.soildyn.2019.106004. [12]R. Dobry, "Simplified methods in Soil Dynamics," Soil Dynamics and Earthquake Engineering, vol. 61-62, pp. 246-268, Jun. 2014, doi: 10.1016/j.soildyn.2014.02.008. 
[13]R. W. Boulanger, C. J. Curras, B. L. Kutter, D. W. Wilson, and A. Abghari, "Seismic Soil-PileStructure Interaction Experiments and Analyses," Journal of Geotechnical and Geoenvironmental Engineering, vol. 125, no. 9, pp. 750-759, Sep. 1999, doi: 10.1061/(ASCE)10900241(1999)125:9(750).

[14]P. Raychowdhury and T. C. Hutchinson, "Performance evaluation of a nonlinear Winkler-based shallow foundation model using centrifuge test results," Earthquake Engng Struct. Dyn., vol. 38, no. 5, pp. 679-698, Apr. 2009, doi: 10.1002/eqe.902.

[15]L. Bhaumik and P. Raychowdhury, "Seismic response analysis of a nuclear reactor structure considering nonlinear soil-structure interaction," Nuclear Engineering and Design, vol. 265, pp. 1078-1090, Dec. 2013, doi: 10.1016/j.nucengdes.2013.09.037.

[16]R. Siddharthan, S. Ara, and G. M. Norris, "Simple Rigid Plastic Model for Seismic Tilting of Rigid Walls," Journal of Structural Engineering, vol. 118, no. 2, pp. 469-487, Feb. 1992, doi: 10.1061/(ASCE)0733-9445(1992)118:2(469).

[17]N. Allotey and M. H. El Naggar, "An investigation into the Winkler modeling of the cyclic response of rigid footings," Soil Dynamics and Earthquake Engineering, vol. 28, no. 1, pp. 44-57, Jan. 2008, doi: 10.1016/j.soildyn.2007.04.003.

[18]G. T. Houlsby, M. J. Cassidy, and I. Einav, "A generalised Winkler model for the behaviour of shallow foundations," Géotechnique, vol. 55, no. 6, pp. 449-460, Aug. 2005, doi:

10.1680/geot.2005.55.6.449.

[19]G. Gazetas, I. Anastasopoulos, O. Adamidis, and Th. Kontoroupi, "Nonlinear rocking stiffness of foundations," Soil Dynamics and Earthquake Engineering, vol. 47, pp. 83-91, Apr. 2013, doi: 10.1016/j.soildyn.2012.12.011.

[20]I. Anastasopoulos and Th. Kontoroupi, "Simplified approximate method for analysis of rocking systems accounting for soil inelasticity and foundation uplifting," Soil Dynamics and Earthquake Engineering, vol. 56, pp. 28-43, Jan. 2014, doi: 10.1016/j.soildyn.2013.10.001.

[21]I. Anastasopoulos, L. Sakellariadis, and A. Agalianos, "Seismic analysis of motorway bridges accounting for key structural components and nonlinear soil-structure interaction," Soil Dynamics and Earthquake Engineering, vol. 78, pp. 127-141, Nov. 2015, doi: 10.1016/j.soildyn.2015.06.016. [22]Y. Lu, A. M. Marshall, and I. Hajirasouliha, "A simplified Nonlinear Sway-Rocking model for evaluation of seismic response of structures on shallow foundations," Soil Dynamics and Earthquake Engineering, vol. 81, pp. 14-26, Feb. 2016, doi: 10.1016/j.soildyn.2015.11.002. [23]Z. Li, S. Escoffier, and P. Kotronis, "Study on the stiffness degradation and damping of pile foundations under dynamic loadings," Engineering Structures, vol. 203, p. 109850, Jan. 2020, doi: 10.1016/i.engstruct.2019.109850.

[24]E. Ahmadi, M. Kiani, F. Paytam, and F. Khoshnoudian, "Equivalent linearization parameters of soil-MDOF structure systems subjected to pulse-like earthquakes," Soils and Foundations, vol. 58, no. 6, pp. 1371-1382, Dec. 2018, doi: 10.1016/j.sandf.2018.08.006.

[25]M. Vucetic and A. Mortezaie, "Cyclic secant shear modulus versus pore water pressure in sands at small cyclic strains," Soil Dynamics and Earthquake Engineering, vol. 70, pp. 60-72, Mar. 2015, doi: 10.1016/j.soildyn.2014.12.001.

[26]W. D. Iwan, "A Distributed-Element Model for Hysteresis and Its Steady-State Dynamic Response," Journal of Applied Mechanics, vol. 33, no. 4, pp. 893-900, Dec. 1966, doi: 10.1115/1.3625199.

[27]P. D. Spanos, "Hysteretic structural vibrations under random load," The Journal of the Acoustical Society of America, vol. 65, no. 2, pp. 404-410, Feb. 1979, doi: 10.1121/1.382338. [28]F. Preisach, "Uber die magnetische Nachwirkung," Z. Physik, vol. 94, no. 5-6, pp. 277-302, May 1935, doi: 10.1007/BF01349418. 
[29]M. A. Krasnosel'skiı̌ and A. V. Pokrovskiı̌, Systems with hysteresis. Berlin; New York: Springer-Verlag, 1989.

[30]I. D. Mayergoyz, Mathematical models of hysteresis. Place of publication not identified: Springer, 2012.

[31] R. Fares, M.P. Santisi D'avila, A. Deschamps. Soil-structure interaction analysis using a 1DT3C wave propagation model. Soil Dynamics and Earthquake Engineering, Elsevier, 2019, 120, pp.200-213.

[32]G. Gazetas and G. Mylonakis, "Seismic soil-structure interaction: New evidence and emerging issues," vol. Geotechnical Special Publication, no. 75 II, pp. 1119-1174, 1998.

[33]P. D. Spanos, P. Cacciola, and G. Muscolino, "Stochastic Averaging of Preisach Hysteretic Systems," J. Eng. Mech., vol. 130, no. 11, pp. 1257-1267, Nov. 2004, doi: 10.1061/(ASCE)07339399(2004)130:11(1257).

[34]P. D. Spanos, A. Kontsos, and P. Cacciola, "Steady-State Dynamic Response of Preisach Hysteretic Systems," Journal of Vibration and Acoustics, vol. 128, no. 2, pp. 244-250, Apr. 2006, doi: $10.1115 / 1.2159041$.

[35]A. S. Veletsos and J. W. Meek, "Dynamic behaviour of building-foundation systems," Earthquake Engng. Struct. Dyn., vol. 3, no. 2, pp. 121-138, 1974, doi: 10.1002/eqe.4290030203. [36]P. D. Spanos, P. Cacciola, and J. Redhorse, "Random Vibration of SMA Systems via Preisach Formalism," Nonlinear Dynamics, vol. 36, no. 2-4, pp. 405-419, Jun. 2004, doi:

10.1023/B:NODY.0000045514.54248.fa.

[37]T. Doong and I. Mayergoyz, "On numerical implementation of hysteresis models," IEEE Trans. Magn., vol. 21, no. 5, pp. 1853-1855, Sep. 1985, doi: 10.1109/TMAG.1985.1063923.

[38]A. V. Lubarda, D. Sumarac, and D. Krajcinovic, "Hysteretic response of ductile materials subjected to cyclic loads," in Recent Advances in Damage Mechanics and Plasticity, 1992, vol. 123, pp. $145-157$.

[39]A. Ktena, D. I. Fotiadis, P. D. Spanos, and C. V. Massalas, “A Preisach model identification procedure and simulation of hysteresis in ferromagnets and shape-memory alloys," Physica B:

Condensed Matter, vol. 306, no. 1-4, pp. 84-90, Dec. 2001, doi: 10.1016/S0921-4526(01)00983-8. [40]A. Ktena, D. I. Fotiadis, P. D. Spanos, A. Berger, and C. V. Massalas, "Identification of 1D and 2D Preisach models for ferromagnets and shape memory alloys," International Journal of Engineering Science, vol. 40, no. 20, pp. 2235-2247, Dec. 2002, doi: 10.1016/S00207225(02)00116-7.

[41] G M Jenkins, Analysis of the stress-strain relationships in reactor grade graphite, Br. J. Appl. Phys. 13, 30, 1962.

[42] W. D Iwan., and P.-T. D Spanos, "Response envelope statistics for nonlinear oscillators with random excitation." J. Appl. Mech., 45, 170-178, 1978

[43]S. L. Kramer, Geotechnical earthquake engineering. Upper Saddle River, N.J.: Prentice Hall, 1996.

[44]D. J. Malcolm and A. C. Hansen, "WindPACT Turbine Rotor Design Study,” National Renewable Energy Laboratory, 2002.

[45]F. Taddei and K. Meskouris, "Seismic Analysis of Onshore Wind Turbine Including SoilStructure Interaction Effects," in Seismic Design of Industrial Facilities, S. Klinkel, C. Butenweg, G. Lin, and B. Holtschoppen, Eds. Wiesbaden: Springer Fachmedien Wiesbaden, 2014, pp. 511522.

[46]K. H. Andersen, A. Kleven, and D. Heien, "Cyclic Soil Data for Design of Gravity Structures," Journal of Geotechnical Engineering, vol. 114, no. 5, pp. 517-539, May 1988, doi: 10.1061/(ASCE)0733-9410(1988)114:5(517). 
[47]Det Norske Veritas and Forskningscenter Risø, Eds., Guidelines for design of wind turbines, 2. ed. Copenhagen: Det Norske Veritas [u.a.], 2009.

[48]S. Kontoe, L. Zdravkovic, and D. M. Potts, "An assessment of time integration schemes for dynamic geotechnical problems," Computers and Geotechnics, vol. 35, no. 2, pp. 253-264, Mar. 2008, doi: 10.1016/j.compgeo.2007.05.001. 